\title{
Design Sensitivity Analysis and Optimization of High Frequency Radiation Problems Using Energy Finite Element Method and Energy Boundary Element Method
}

\author{
Kyung K. Choi ${ }^{*}$ and Jun Dong ${ }^{\dagger}$ \\ Department of Mechanical and Industrial Engineering and Center for Computer-Aided Design, University of Iowa, \\ Iowa City, IA 52242, USA \\ Nickolas Vlahopoulos ${ }^{\ddagger}$, Aimin Wang ${ }^{\S}$, Weiguo Zhang ${ }^{* *}$ \\ Department of Naval Architecture and Marine Engineering, University of Michigan, Ann Arbor, MI 48109, USA
}

This paper presents a continuum design sensitivity analysis (DSA) and optimization of high frequency radiation problems using the Energy Finite Element Method (EFEM) and Energy Boundary Element Method (EBEM). The noise radiated from the vibrating structure at a high frequency range is obtained through a sequential procedure. The structural EFEM calculates structural energy distribution, which is then used as the boundary condition for EBEM to calculate the energy density at a far-field observation point. For DSA, the direct differentiation method calculates the sensitivity of the exterior noise through the sensitivity of the structural energy density obtained from EFEM. The adjoint variable method calculates the adjoint load from an acoustic EBEM re-analysis, and the adjoint response is obtained from a structural EFEM re-analysis. The sensitivity information is obtained by carrying out numerical integration only on the structural FE part. The proposed DSA approach has been applied in the design of automotive and naval structures to search for the best material layout to achieve lowest noise level at high frequency.

\section{Nomenclature}

$\begin{array}{ll}\boldsymbol{x} & =\text { Position vector } \\ \rho_{s} & =\text { Structural mass density } \\ p & =\text { Acoustic pressure } \\ \rho_{0} & =\text { Acoustic mass density } \\ c_{g} & =\text { Group speed } \\ c_{0} & =\text { Acoustic wave speed } \\ h & =\text { Thickness of plate } \\ f & =\text { Frequency } \\ \omega & =\text { Radian frequency } \\ \psi & =\text { Performance Measure } \\ \boldsymbol{u} & =\text { Design variable vector } \\ \varepsilon & =\text { Perturbation size } \\ \delta \boldsymbol{u} & =\text { Design perturbation direction } \\ e & =\text { Energy density } \\ e^{\prime} & =\text { Partial derivative of energy density }\end{array}$

\footnotetext{
* Professor and Corresponding Author, kkchoi@ccad.uiowa.edu, AIAA Associate Fellow

† Graduate Research Assistant, jundong@ccad.uiowa.edu

*Associate Professor, nickvl@engin.umich.edu

§esearch Associate, wangam@engin.umich.edu

** Graduate Research Assistant, zhangwg@engin.umich.edu
} 


$\begin{array}{ll}c_{g B} & =\text { Structural bending group speed } \\ \eta & =\text { Hysteresis damping factor } \\ \pi & =\text { Power density input } \\ k_{s B} & =\text { Structural bending wave number } \\ k & =\text { Acoustic wave number } \\ \sigma_{\text {rad }} & =\text { Radiation efficiency } \\ \eta_{\text {rad }} & =\text { Radiation damping } \\ \alpha & =\text { Effective mass density coefficient } \\ \boldsymbol{I} & =\text { Energy intensity } \\ P & =\text { Power radiation } \\ a_{u}(\cdot, \bullet) & =\text { Structural energy bilinear form } \\ b_{u}(\bullet, \bullet) & =\text { Structural-structural coupling term } \\ \ell_{u}(\bullet) & =\text { Structural load linear form } \\ \boldsymbol{K} & =\text { Structural stiffness matrix } \\ \boldsymbol{J}_{s s} & =\text { Structural-structural coupling matrix } \\ \tau & =\text { Power transfer coefficient } \\ \lambda_{s} & =\text { Structural adjoint response vector } \\ \boldsymbol{\xi} & =\text { Acoustic adjoint load vector (for energy density) } \\ \boldsymbol{\zeta} & =\text { Acoustic adjoint load vectors (for energy intensity) } \\ \boldsymbol{F}_{a d j} & =\text { Structural adjoint load } \\ \boldsymbol{G}\left(\boldsymbol{x}, \boldsymbol{x}_{0}\right) & =\text { Green's function for time-averaged energy density } \\ \boldsymbol{H}\left(\boldsymbol{x}, \boldsymbol{x}_{0}\right) & =\text { Green's function for time-averaged energy intensity } \\ s(\bullet) & =\text { Linear scalar integral form } \\ \boldsymbol{m}(\bullet) & =\text { Linear vector integral form } \\ \boldsymbol{H} & =\text { Stiffness matrix of EBEM } \\ \boldsymbol{\sigma} & =\text { Acoustic energy strength vector } \\ \boldsymbol{W} & =\text { Conversion matrix } \\ h(\bullet) & =\text { Linear integral form } \\ w(\bullet) & =\text { Linear integral form } \\ & \end{array}$

\section{Introduction}

The objective of this paper is to present an analytical approach for the design sensitivity analysis of structureinduced sound and vibration at a high frequency range to design structural systems with desirable acoustic performance while minimizing the mass of the structure. To this end, the Energy Finite Element Method (EFEM) and Energy Boundary Element Method (EBEM) are used to predict the noise radiated from a vibrating structure and continuum design sensitivity analysis is developed for a sequential EFEM-EBEM procedure for parametric design variables, such as the thickness and material property of a structural plate to evaluate design sensitivity information at the current design and study the potential design change for an optimum design.

The reason that the EFEM and EBEM are used for high frequency structural-acoustic analysis over the traditional Finite Element Method (FEM) [1-3] and Boundary Element Method (BEM) [4-6] is because that the FEM and BEM are proved to be only suitable for structural-acoustic problems at low frequency range. Since the structural or acoustic domain is discretized in finite elements or boundary elements for FEM or BEM, the element size should be small enough to accurately capture the wave propagation through the structural or acoustic domain. Atalla and Bernhard [7] presented a complete comparison of FEM and BEM in low frequency structural-acoustic analysis, and they recommended that at least six linear elements or three quadratic elements be required per wavelength for an accurate analysis. From their research, it is commonly agreed that FEM and BEM would only be suitable for a given problem with a maximum frequency of $200 \mathrm{~Hz}$.

Statistical Energy Analysis (SEA) is an alternative to analyze the response of vibrational structures [8-10] at high frequency. According to SEA, the structural system is divided into subsystems with similar energy modes and the power balance equation is solved to obtain the lumped energy carried by each subsystem. The biggest drawback for 
SEA is that the variation of energy response cannot be predicted and design variables do not appear in the governing equation of SEA, so it is not a preferred analysis tool from a design point of view.

The Energy Finite Element Method (EFEM) is the application of finite element techniques in power balance equation to obtain a localized time- and space-averaged energy density solution [11-20]. Since energy conservation is imposed locally in power flow analysis, it is possible to represent the structural geometry in detail, which is critical from a design point of view. The response variable (energy density) is not continuous across structural junctions and structural-acoustic interfaces in EFEM. In order to assemble different structural components (rod, beam, membrane, plate, etc.) and to place an acoustic medium into built-up structures, a coupling matrix method was developed [16,17] in which the structural-structural and structural-acoustic power transfer coefficients $[20,21]$ are used to consider power flow conservation between coupled structural components and structural-acoustic interfaces. The effect of fluid loading on structural vibration at a high frequency is investigated by Zhang et al. [22] and as a result, the power flow governing differential equation is modified to consider the fluid effect and the application of EFEM is extended to vibrating structure immersed in dense fluid, i.e., naval engineering.

Energy finite element method has been successfully applied to various engineering problems. Vlahopoulos et al. $[23,24]$ validated EFEM by applying it to a complex ship structure and comparing the EFEM results to SEA and a very refined FEM results. Wang [25] used a degenerated version of EFEM to analyze the cabin of a heavy-duty truck.

Since the EFEM still requires the discretization of the 3-D acoustic domain, it is not a favorite choice for radiation analysis where the domain is infinite. For the high frequency radiation problem, Wang et al. [26] developed an energy boundary element formulation using an acoustic energy corollary. The boundary condition of the Energy Boundary Element Method (EBEM), which is energy intensity on the structural surface, can be obtained either from a structural energy finite element analysis, or by being measured through experiments. This method is applied in the analysis of the radiation of an undersea ship in water [26]. The EFEM and EBEM constitute a sequential procedure: a structural EFEM solves the structural energy density and the power radiation from the structure, which will be used as a boundary condition of EBEM to compute the radiated far-file noise.

In contrast to the many research efforts in the design sensitivity analysis (DSA) of low frequency structuralacoustic problems using FEM and BEM [27-38], DSA of high frequency structural-acoustic problems has not been touched until Kim et al. [39] proposed a design sensitivity formulation for structural systems using EFEM. In Kim's research, the structural-acoustic relationship was not addressed until Choi and Dong developed detailed derivations of the parametric design sensitivity formulation for the structural-acoustic coupled problem [40,41].

Using the DSA formulation developed by Choi and Dong for EFEM [40,41], the high frequency radiation noise calculated by a sequential EFEM-EBEM procedure can be obtained from either a direct differentiation or an adjoint variable method: the direct differentiation method calculates the sensitivity of the radiation noise through the sensitivity of the structural energy density obtained from EFEM. The adjoint variable method calculates the adjoint load from an acoustic EBEM re-analysis, and the adjoint response is obtained from a structural EFEM re-analysis. The sensitivity information is obtained by carrying out numerical integration only on the structural FE part. Both methods present excellent numerical accuracy as well as computational efficiency compared with the finite difference approaches.

\section{Theoretical Background}

\section{Energy Finite Element Method}

The governing equation of EFEM is derived for the time- and space- averaged energy density $e$ as [11-20]

$$
-\frac{c_{g}^{2}}{\eta \omega} \nabla^{2} e+\eta \omega e=\pi
$$

In the governing equation (1), $\eta$ is the local material hysteresis damping factor, $c_{g}$ is the group speed, and $\omega$ is the excitation radian frequency. On the right side of the equation, $\pi$ is the power density input to the system. The first term on the left side represents the transmitted power from the local system to the outside and adjacent systems, whereas the second term on the left side represents the time- and space- averaged dissipated power inside the local system. Equation (1) explains energy conservation in a local system: energy flow into the structure is equal to the energy that dissipates in the structure plus the energy flow across the boundaries. This formula can be applied to both structural and acoustic wave motion in similar forms. For a complex structural-acoustic system, the energy density $e$ is not continuous on the geometric and material discontinuities. However, the energy flow remains 
continuous on the structural-structural junctions and structural-acoustic interfaces. If the interior acoustic domain is ignored in a radiation analysis, a variational equation can be derived for the structural part from governing Eq. (1) using the energy conservation law as

$$
a_{u}\left(\boldsymbol{e}_{s}, \overline{\boldsymbol{e}}_{s}\right)+b_{u}\left(\boldsymbol{e}_{s}, \overline{\boldsymbol{e}}_{s}\right)=\ell_{u}\left(\overline{\boldsymbol{e}}_{s}\right), \quad \forall \overline{\boldsymbol{e}}_{s} \in Z_{s}
$$

In Eq. (2), the subscript " $s$ " is used to indicate the energy density on the structural domain, $Z_{s}$ is the kinematically admissible energy space, and the subscript " $u$ " indicates the design dependency of the energy terms. Among the energy terms, $a_{u}(\bullet, \bullet)$ is the energy bilinear form, $\ell_{u}(\bullet)$ is the load linear form, and $b_{u}(\bullet, \bullet)$ is the coupling term between structural sub-domains. The coupling term is difficult to express in continuum form, but a structuralstructural coupling matrix $\boldsymbol{J}_{s s}$ can be constructed to express $b(\cdot, \bullet)$ in discrete form as

$$
b_{u}\left(\boldsymbol{e}_{s}, \overline{\boldsymbol{e}}_{s}\right)=\left\{\overline{\boldsymbol{E}}_{s}\right\}^{T}\left[\boldsymbol{J}_{s s}\right]\left\{\boldsymbol{E}_{s}\right\}
$$

Discretization of the variational Eq. (2) leads to the system equation of EFEM as

$$
\left[\boldsymbol{K}_{s}+\boldsymbol{J}_{s s}\right]\left\{\boldsymbol{E}_{s}\right\}=\left\{\boldsymbol{F}_{s}\right\}
$$

Solution of Eq. (4) yields $\boldsymbol{E}_{s}$, the nodal energy density vector on the structure. Since the coupling matrices $\boldsymbol{J}_{s s}$ is constructed from structural-structural power transfer coefficients, which are un-symmetric due to the power transfer natures between structural components, the system equation is un-symmetric. The expressions of the energy bilinear form $a_{u}(\bullet, \bullet)$, coupling term $b_{u}(\bullet, \bullet)$, the load linear form $\ell_{u}(\bullet)$ and the expressions of the structural stiffness matrices $\boldsymbol{K}_{s}$ and structural-structural coupling matrix $\boldsymbol{J}_{s s}$ can be found in Choi and Dong [40, 41].

\section{Energy Boundary Element Method}

The basic integral equation of the EBEM formulation is derived from the acoustic energy corollary [26]. Consider an acoustic medium with volume $V$ which encloses some acoustic energy sources, the area surrounding the volume $V$ is $S$ as shown in Fig. 1, and $\boldsymbol{I}$ is the acoustic energy intensity on the boundary $S$ in the acoustic medium, then the total time-averaged acoustic power $P$ radiated by the energy sources can be obtained by the following integral equation:

$$
P=\int_{S} \boldsymbol{I} \cdot \boldsymbol{n} d S
$$

where $\boldsymbol{n}$ is the unit normal vector pointing out of the volume.

Consider a spherical energy source with strength $\sigma$ located at a field point $\boldsymbol{x}$, the time-averaged energy density and the time-averaged energy intensity at an arbitrary field point $x_{0}$ due to this acoustic energy source can be calculated by the following equation

$$
\begin{gathered}
e\left(\boldsymbol{x}_{0}\right)=G\left(\boldsymbol{x}, \boldsymbol{x}_{0}\right) \sigma \\
\boldsymbol{I}\left(\boldsymbol{x}_{0}\right)=H\left(\boldsymbol{x}, \boldsymbol{x}_{0}\right) \boldsymbol{u}\left(\boldsymbol{x}, \boldsymbol{x}_{0}\right) \sigma
\end{gathered}
$$

where $\boldsymbol{u}\left(\boldsymbol{x}, \boldsymbol{x}_{0}\right)$ is the unit normal vector pointing from point $\boldsymbol{x}$ to $\boldsymbol{x}_{0} ; \boldsymbol{G}\left(\boldsymbol{x}, \boldsymbol{x}_{0}\right)$ and $\boldsymbol{H}\left(\boldsymbol{x}, \boldsymbol{x}_{0}\right)$ are the corresponding Green's functions for the time-averaged acoustic energy density and the time-averaged acoustic energy intensity in the free field, and

$$
G\left(\boldsymbol{x}, \boldsymbol{x}_{0}\right)=\frac{\rho_{0}}{64 \pi^{2} r^{4}\left(\boldsymbol{x}, \boldsymbol{x}_{0}\right)}+\frac{k^{2} \rho_{0}}{32 \pi^{2} r^{2}\left(\boldsymbol{x}, \boldsymbol{x}_{0}\right)}
$$




$$
H\left(\boldsymbol{x}, \boldsymbol{x}_{0}\right)=\frac{k^{2} \rho_{0} c_{0}}{32 \pi^{2} r^{2}\left(\boldsymbol{x}, \boldsymbol{x}_{0}\right)}
$$

where $c_{0}$ is the air speed in the acoustic medium, $\rho_{0}$ is the acoustic mass density, $k$ is the acoustic wave number, and $r\left(\boldsymbol{x}, \boldsymbol{x}_{0}\right)$ is the distance from the acoustic energy source to the field point.

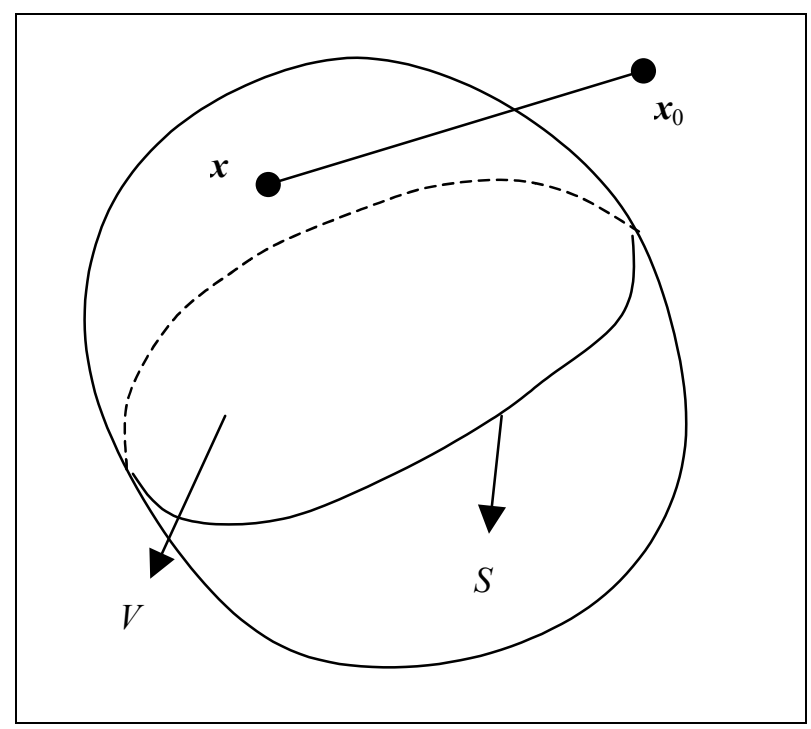

Figure 1 Arbitrary Volume $V$ Bounded by Surface $S$

The above equation can be extended from a single energy source to a group of incoherent acoustic energy sources, which then constitutes the governing equation of EBEM. For an arbitrary structure bounded by a surface $S$ in an infinite acoustic medium, if the surface can be divided into quadrilateral and triangular elements and each element acts as an incoherent acoustic energy source with strength $\sigma_{j}(j=1,2,3, N B E)$, then the time-averaged acoustic energy density and acoustic energy intensity at a general field point $\boldsymbol{x}_{0}$ in the space outside of the structure can be calculated by the superposition of the Eqs. (8) and (9) for each energy source as

$$
\begin{gathered}
e\left(\boldsymbol{x}_{0}\right)=\sum_{j=1}^{N B E} \int_{S_{j}} G\left(\boldsymbol{y}, \boldsymbol{x}_{0}\right) \frac{\sigma_{j}}{A_{j}} d S=\sum_{j=1}^{N B E} \frac{\sigma_{j}}{A_{j}} \int_{S_{j}} G\left(\boldsymbol{y}, \boldsymbol{x}_{0}\right) d S \\
\boldsymbol{I}\left(\boldsymbol{x}_{0}\right)=\sum_{j=1}^{N B E} \int_{S_{j}} H\left(\boldsymbol{y}, \boldsymbol{x}_{0}\right) \boldsymbol{u}\left(\boldsymbol{y}, \boldsymbol{x}_{0}\right) \frac{\sigma_{j}}{A_{j}} d S=\sum_{j=1}^{N B E} \frac{\sigma_{j}}{A_{j}} \int_{S_{j}} H\left(\boldsymbol{y}, \boldsymbol{x}_{0}\right) \boldsymbol{u}\left(\boldsymbol{y}, \boldsymbol{x}_{0}\right) d S
\end{gathered}
$$

In above equations, $N B E$ is the number of elements on the surface, $A_{j}$ is the area of the $j$-th element, $\boldsymbol{y}$ is an arbitrary point on the $j$-th element, $\boldsymbol{u}\left(\boldsymbol{y}, \boldsymbol{x}_{0}\right)$ is the unit normal vector pointing from point $\boldsymbol{y}$ to the field point $\boldsymbol{x}_{0}$. Since the energy strength $\sigma_{j}$ is supposed to be constant on a given element, it can be brought outside of the integral with the area $A_{j}$. The above equations can be written in continuum forms as

$$
\begin{aligned}
& e\left(\boldsymbol{x}_{0}\right)=s\left(\boldsymbol{x}_{0} ; \boldsymbol{\sigma}\right) \\
& \boldsymbol{I}\left(\boldsymbol{x}_{0}\right)=\boldsymbol{m}\left(\boldsymbol{x}_{0} ; \boldsymbol{\sigma}\right)
\end{aligned}
$$


where $s(\bullet)$ and $\boldsymbol{m}(\bullet)$ are linear scalar and vector integral forms in terms of the acoustic energy strength respectively.

If the field point $\boldsymbol{x}_{0}$ is located on the surface, then Eq. (11) can be used to calculate the acoustic energy intensity of each element on the surface. For example, if the field point $\boldsymbol{x}_{0}$ is evaluated at an arbitrary point $\boldsymbol{z}$ inside the $i$-th element, the energy intensity at this point $\boldsymbol{z}$ can be calculated by substituting $\boldsymbol{x}_{0}$ in Eq. (11) by $\boldsymbol{z}$ as

$$
\boldsymbol{I}(\boldsymbol{z})=\sum_{j=1}^{N B E} \frac{\sigma_{j}}{A_{j}} \int_{S_{j}} H(\boldsymbol{y}, \boldsymbol{z}) \boldsymbol{u}(\boldsymbol{y}, \boldsymbol{z}) d S
$$

Once the energy intensity of an acoustic energy source $\sigma_{i}$ of the $i$-th element on the surface is known, the acoustic power radiated from this energy source into the acoustic medium can be obtained from Eq. (5) as

$$
\int_{S_{i}} \boldsymbol{I}_{i} \cdot \boldsymbol{n}_{i} d S=P_{i}, \quad(i=1,2, \cdots, N B E)
$$

where $P_{i}$ is the acoustic power radiated by the $i$-th element. Substitution of Eq. (14) into Eq. (15) leads to

$$
\int_{S_{i}}\left[\sum_{j=1}^{N B E} \frac{\sigma_{j}}{A_{j}} \int_{S_{j}} H(\boldsymbol{y}, \boldsymbol{z}) \boldsymbol{u}(\boldsymbol{y}, \boldsymbol{z}) d S\right] \cdot \boldsymbol{n}_{i} d S=P_{i}, \quad(i=1,2, \cdots, N B E)
$$

If we write the acoustic power $P$ radiated from each element and the acoustic energy strength $\sigma$ of each element in vector forms as

$$
\begin{gathered}
\boldsymbol{P}=\left\{\begin{array}{llll}
P_{1} & P_{2} & \cdots & P_{N B E}
\end{array}\right\}^{T} \\
\boldsymbol{\sigma}=\left\{\begin{array}{llll}
\sigma_{1} & \sigma_{2} & \cdots & \sigma_{N B E}
\end{array}\right\}^{T}
\end{gathered}
$$

then Eq. (16) can be rewritten in matrix form as

$$
H \sigma=P
$$

where matrix $\boldsymbol{H}$ is defined as the stiffness matrix of EBEM, and its component is derived from Eq. (16) as

$$
H_{i j}=\int_{S_{i}}\left[\int_{S_{j}} \frac{H(\boldsymbol{y}, \boldsymbol{z}) \boldsymbol{u}(\boldsymbol{y}, \boldsymbol{z})}{A_{j}} d S\right] \cdot \boldsymbol{n}_{i} d S
$$

The numerical evaluation of $H_{i j}$ can be obtained by applying Gaussian Quadrature in Eq. (20). However, the diagonal terms of matrix $\boldsymbol{H}$ when $i=j$ can not be computed by the above equation since there is a singularity occurring in the Green's function in Eq. (9) when $r\left(\boldsymbol{x}, \boldsymbol{x}_{0}\right)=0$. In order to overcome this singularity, Wang [26] pointed out that for a spherical energy source with strength $\sigma$, the power radiated by the energy source could be calculated by

$$
\Pi=\frac{k^{2} \rho_{0} c_{0}}{8 \pi} \sigma_{i}
$$

The power associated with the acoustic energy source distributed over an element through one side of the element surface is one-half of the total power П. Therefore, Eq. (15) can be rewritten as

$$
\int_{S_{i}} \boldsymbol{I}_{i} \cdot \boldsymbol{n}_{i} d S=\frac{k^{2} \rho_{0} c_{0}}{16 \pi} \sigma_{i}
$$


As a result, the diagonal term of the stiffness matrix can be calculated by

$$
H_{i i}=\frac{k^{2} \rho_{0} c_{0}}{16 \pi}
$$

In order to solve the acoustic energy strength in Eq. (19), the acoustic power radiated from each element needs to be identified from EFEM analysis. As discussed in the previous chapters, the solution of the EFEM will yield the energy density on the structural domain. Therefore, the acoustic power radiated from the structure to the acoustic medium can be obtained by

$$
P_{i}=\int_{S_{i}} \eta_{r a d} \omega e_{s B} h d S
$$

where $\eta_{\text {rad }}$ is the radiation damping, which is a function of the structural mass density, panel thickness and geometry and the acoustic wave number $k$, and can be calculated by

$$
\eta_{\text {rad }}=\frac{\rho_{0}}{\rho_{s}} \frac{1}{\alpha k h} \sigma_{r a d}
$$

The parameter $\alpha$ is added to the structural mass density $\rho_{s}$ to represent the effective mass density effect, which can be calculated as [22]

$$
\alpha= \begin{cases}1 & f>f_{c} \\ 1+\frac{\rho_{0}}{\rho_{s} h \sqrt{k_{s B}^{2}-k^{2}}}, & f<f_{c}\end{cases}
$$

where $f_{c}$ is defined as the coincidence frequency at which the structural bending wave number $k_{s B}$ coincides with the acoustic wave number $k$. In Eq. (25), $\sigma_{\text {rad }}$ is the radiation efficiency, which quantifies the interaction between the structural bending wave and the acoustic wave, and can be calculated by [42]:

$$
\sigma_{\text {rad }}= \begin{cases}\frac{a+b}{\pi \mu k a b\left(\mu^{2}-1\right)^{\frac{1}{2}}}\left\{\ln \left(\frac{\mu+1}{\mu-1}\right)+\frac{2 \mu}{\mu^{2}-1}\right\} & f<f_{c} \\ \sqrt{\frac{a}{\lambda_{c}}\left(1+\frac{1}{\sqrt{r}}\right)} & f \approx f_{c} \\ \left(1-\frac{f_{c}}{f}\right)^{-\frac{1}{2}} & f>f_{c}\end{cases}
$$

where $r=a / b$ is the ratio between the characteristic length $a$ and $b$ of the plate, $\mu=k_{f} / k$ is the wave number ratio, and $\lambda_{c}=c / f_{\mathrm{c}}$ is the acoustic wavelength at the coincidence frequency.

A conversion matrix $\boldsymbol{W}$ between the nodal bending energy density $\boldsymbol{E}_{s B}$ on the structure and the acoustic power $\boldsymbol{P}$ radiated on each structural element can be established as

$$
\boldsymbol{P}=\boldsymbol{W} \boldsymbol{E}_{s B}
$$

where the component of the conversion matrix is calculated by

$$
W_{i j}=\int_{S_{i}} \Delta_{i j} \eta_{\text {rad }}^{i} \omega h d S
$$


with $\Delta_{i j}=1$ when node $j$ is located on the $i$-th boundary element, and $\Delta_{i j}=0$ when node $j$ is not on the $i$-th boundary element.

Combining Eq. (19) with Eq. (28), the governing equation of EBEM can be set up as

$$
\boldsymbol{H} \boldsymbol{\sigma}=\boldsymbol{W} \boldsymbol{E}_{s B}
$$

or in continuum form as

$$
h(\boldsymbol{x} ; \boldsymbol{\sigma})=w\left(\boldsymbol{x} ; \boldsymbol{e}_{s B}\right)
$$

where $h(\bullet)$ and $w(\bullet)$ are linear integral form in terms of the acoustic energy strength and the structural bending energy density respectively. The solution of Eqs. (30) or (31) then leads to the acoustic source strength for each element on the surface. Therefore, Eqs. (10) and (11) can be readily applied to compute the time-averaged energy density and energy intensity for an arbitrary field point in the free field. Finally, the time-averaged acoustic energy density and energy intensity can be expressed in discrete forms, respectively, as

$$
\begin{aligned}
& e\left(\boldsymbol{x}_{0}\right)=\boldsymbol{S}^{T} \boldsymbol{\sigma} \\
& \boldsymbol{I}\left(\boldsymbol{x}_{0}\right)=\boldsymbol{M} \boldsymbol{\sigma}
\end{aligned}
$$

and the components of vector $\boldsymbol{S}$ and matrix $\boldsymbol{M}$ are derived from Eqs. (10) and (11) as

$$
\begin{gathered}
S_{i}=\frac{1}{A_{i}} \int_{S_{i}} G\left(\boldsymbol{y}, \boldsymbol{x}_{0}\right) d S \\
M_{i j}=\frac{1}{A_{i}} \int_{S_{i}} H\left(\boldsymbol{y}, \boldsymbol{x}_{0}\right) u_{j}\left(\boldsymbol{y}, \boldsymbol{x}_{0}\right) d S \quad(j=1,2,3)
\end{gathered}
$$

It is shown that the EFEM-EBEM constitutes a sequential numerical procedure, where the output of EFEM is used to compute the boundary condition for the EBEM. The EBEM is divided into two steps: the first step is to construct the boundary condition using the results from EFEM and solve the acoustic energy strength on the surface; the second step is to calculate the acoustic energy density and acoustic energy intensity in the far-field point in the infinite acoustic domain.

\section{Design Sensitivity Analysis}

\section{Direct Differentiation Method}

For a given structural-acoustic performance measure which can be written in a integral form of the timeaveraged acoustic energy density $e$ and acoustic energy intensity $\boldsymbol{I}$ in the far-field point as the following

$$
\psi=\int_{\Omega} g(\boldsymbol{u}, e, \nabla e, \boldsymbol{I}, \nabla \boldsymbol{I}) d \Omega
$$

its variation with respect to the design variable $\boldsymbol{u}$ can be obtained as

$$
\psi^{\prime}=\int_{\Omega}\left[g_{, u} \delta \boldsymbol{u}+g_{, e} e^{\prime}+g_{\nabla e} \cdot \nabla e^{\prime}+g_{, \boldsymbol{I}} \cdot \boldsymbol{I}^{\prime}+g_{, \nabla \boldsymbol{I}}: \nabla \boldsymbol{I}^{\prime}\right] d \Omega
$$

where the first-order variation of the energy density $e$ is defined as

$$
e^{\prime}=e^{\prime}(\boldsymbol{x} ; \boldsymbol{u}, \delta \boldsymbol{u})=\left.\frac{d}{d \varepsilon} e(\boldsymbol{x} ; \boldsymbol{u}+\varepsilon \delta \boldsymbol{u})\right|_{\varepsilon=0}=\frac{\partial e^{T}}{\partial \boldsymbol{u}} \delta \boldsymbol{u}
$$


when the design variable $\boldsymbol{u}$ is perturbed in the direction $\delta \boldsymbol{u}$ with the amount $\varepsilon \delta \boldsymbol{u}$. For the structural EFEM, the firstorder variation of the structural energy density $\boldsymbol{e}_{s}$ can be obtained directly from differentiating the variational Eq. (2) with respect to the parametric design variables as

$$
a_{u}\left(\boldsymbol{e}_{s}^{\prime}, \overline{\boldsymbol{e}}_{s}\right)+b_{u}\left(\boldsymbol{e}_{s}^{\prime}, \overline{\boldsymbol{e}}_{s}\right)=\ell_{\delta u}^{\prime}\left(\overline{\boldsymbol{e}}_{s}\right)-a_{\delta u}^{\prime}\left(\boldsymbol{e}_{s}, \overline{\boldsymbol{e}}_{s}\right)-b_{\delta u}^{\prime}\left(\boldsymbol{e}_{s}, \overline{\boldsymbol{e}}_{s}\right), \forall \overline{\boldsymbol{e}}_{s} \in Z_{s}
$$

where the first-order variation of the energy bilinear form $a_{u}(\bullet, \bullet)$, coupling term $b_{u}(\bullet, \bullet)$ and the load linear form $\ell_{u}(\bullet)$ are provided in detail in Choi and Dong [40,41].

Since the integral forms $s(\bullet)$ and $\boldsymbol{m}(\bullet)$ in Eqs. (12) and (13) do not explicitly depend on the structural design, the design sensitivity of the energy density and energy intensity at the exterior field point can be obtained by directly differentiating Eqs. (12) and (13) as

$$
\begin{gathered}
e^{\prime}\left(\boldsymbol{x}_{0}\right)=s\left(\boldsymbol{x}_{0} ; \boldsymbol{\sigma}^{\prime}\right) \\
\boldsymbol{I}^{\prime}\left(\boldsymbol{x}_{0}\right)=\boldsymbol{m}\left(\boldsymbol{x}_{0} ; \boldsymbol{\sigma}^{\prime}\right)
\end{gathered}
$$

where $\sigma^{\prime}$ is the derivative of the acoustic energy strength on the structural boundary elements, and it can be obtained from the differentiation of the governing equation (31) of EBEM as

$$
\boldsymbol{\sigma}^{\prime}=h^{-1} \cdot w\left(\boldsymbol{x} ; \boldsymbol{e}_{s B}^{\prime}\right)+h^{-1} \cdot w_{\delta u}^{\prime}\left(\boldsymbol{x} ; \boldsymbol{e}_{s B}\right)
$$

where $w_{\delta u}^{\prime}(\bullet)$ is the variation of the integral form $w(\bullet)$ with respect to the design variable $\boldsymbol{u}$. For the structural damping design variable, $w_{\delta u}^{\prime}(\cdot)$ vanishes, whereas for the structural thickness design variable, $w_{\delta u}^{\prime}(\bullet)$ is

$$
w_{\delta u}^{\prime}=\int_{S}\left(\eta_{\text {rad }} \omega \delta h+\omega h \delta \eta_{\text {rad }}\right) d S
$$

and the expression of $\delta \eta_{\text {rad }}$ is derived by Choi and Dong [40-41].

Substitute Eqs. (40)-(43) into Eq. (37), the sensitivity of the performance measure can be obtained as

$$
\begin{aligned}
\psi^{\prime}=\int_{\Omega} & {\left[g_{, u} \delta \boldsymbol{u}+g_{, e} s\left(h^{-1} \cdot w\left(\boldsymbol{e}_{s B}^{\prime}\right)+h^{-1} \cdot w_{\delta u}^{\prime}\left(\boldsymbol{e}_{s B}\right)\right)\right.} \\
& +g_{, \nabla e} \cdot \nabla s\left(h^{-1} \cdot w\left(\boldsymbol{e}_{s B}^{\prime}\right)+h^{-1} \cdot w_{\delta u}^{\prime}\left(\boldsymbol{e}_{s B}\right)\right) \\
& +g_{, I} \cdot \boldsymbol{m}\left(h^{-1} \cdot w\left(\boldsymbol{e}_{s B}^{\prime}\right)+h^{-1} \cdot w_{\delta u}^{\prime}\left(\boldsymbol{e}_{s B}\right)\right) \\
& \left.+g_{, \nabla \boldsymbol{I}}: \nabla \boldsymbol{m}\left(h^{-1} \cdot w\left(\boldsymbol{e}_{s B}^{\prime}\right)+h^{-1} \cdot w_{\delta u}^{\prime}\left(\boldsymbol{e}_{s B}\right)\right)\right] d \Omega
\end{aligned}
$$

Equation (44) provides the expression to calculate the sensitivity of the performance measure in terms of the sensitivity of the structural energy density for the direct differentiation method. The solution of the sensitivity of the energy density from EFEM in Eq. (39) then can be substituted into the above equation to get the sensitivity information of an arbitrary structural-acoustic performance measure in Eq. (36).

\section{Adjoint Variable Method}

While the direct differentiation method calculates the sensitivity information through the sensitivity of the structural energy density from EFEM, the adjoint variable method can achieve the same goal by defining an adjoint equation with the adjoint load from the right side of Eq. (44) that are implicitly dependent on the design variables

$$
\begin{aligned}
a_{u}\left(\overline{\boldsymbol{\lambda}}_{s}, \boldsymbol{\lambda}_{s}\right)+b_{u}\left(\overline{\boldsymbol{\lambda}}_{s}, \boldsymbol{\lambda}_{s}\right) & =\int_{\Omega}\left[g_{, e} s\left(h^{-1} \cdot w\left(\overline{\boldsymbol{\lambda}}_{s}\right)\right)+g_{, \nabla e} \cdot \nabla s\left(h^{-1} \cdot w\left(\overline{\boldsymbol{\lambda}}_{s}\right)\right)\right. \\
& \left.+g_{, I} \cdot \boldsymbol{m}\left(h^{-1} \cdot w\left(\overline{\boldsymbol{\lambda}}_{s}\right)\right)+g_{, \nabla I}: \nabla \boldsymbol{m}\left(h^{-1} \cdot w\left(\overline{\boldsymbol{\lambda}}_{s}\right)\right)\right] d \Omega
\end{aligned}, \quad \forall \overline{\boldsymbol{\lambda}}_{s} \in Z_{s}
$$


Evaluation of the above equation at $\bar{\lambda}_{s}=\boldsymbol{e}_{s}^{\prime}$ yields

$$
\begin{aligned}
a_{u}\left(\boldsymbol{e}_{s}^{\prime}, \boldsymbol{\lambda}_{s}\right)+b_{u}\left(\boldsymbol{e}_{s}^{\prime}, \boldsymbol{\lambda}_{s}\right) & =\int_{\Omega}\left[g_{, e} s\left(h^{-1} \cdot w\left(\boldsymbol{e}_{s}^{\prime}\right)\right)+g_{, \nabla e} \cdot \nabla s\left(h^{-1} \cdot w\left(\boldsymbol{e}_{s}^{\prime}\right)\right)\right. \\
& \left.+g_{, I} \cdot \boldsymbol{m}\left(h^{-1} \cdot w\left(\boldsymbol{e}_{s}^{\prime}\right)\right)+g_{, \nabla I}: \nabla \boldsymbol{m}\left(h^{-1} \cdot w\left(\boldsymbol{e}_{s}^{\prime}\right)\right)\right] d \Omega
\end{aligned}
$$

Similarly, because Eq. (39) holds for arbitrary $\overline{\boldsymbol{e}} \in Z_{s}$, it can be evaluated at $\overline{\boldsymbol{e}}_{s}=\lambda_{s}$ to obtain

$$
a_{u}\left(\boldsymbol{e}_{s}^{\prime}, \lambda_{s}\right)+b_{u}\left(\boldsymbol{e}_{s}^{\prime}, \lambda_{s}\right)=\ell_{\delta u}^{\prime}\left(\lambda_{s}\right)-a_{\delta u}^{\prime}\left(\boldsymbol{e}_{s}, \lambda_{s}\right)-b_{\delta u}^{\prime}\left(\boldsymbol{e}_{s}, \lambda_{s}\right)
$$

Since Eqs. (46) and (47) have the same left sides, equating their right sides yields

$$
\begin{aligned}
& \int_{\Omega}\left[g_{, e} s\left(h^{-1} \cdot w\left(\boldsymbol{e}_{s}^{\prime}\right)\right)+g_{, \nabla e} \cdot \nabla s\left(h^{-1} \cdot w\left(\boldsymbol{e}_{s}^{\prime}\right)\right)\right. \\
& \left.+g_{, I} \cdot \boldsymbol{m}\left(h^{-1} \cdot w\left(\boldsymbol{e}_{s}^{\prime}\right)\right)+g_{, \nabla \boldsymbol{I}}: \nabla \boldsymbol{m}\left(h^{-1} \cdot w\left(\boldsymbol{e}_{s}^{\prime}\right)\right)\right] d \Omega \\
& =\ell_{\delta u}^{\prime}\left(\boldsymbol{\lambda}_{s}\right)-a_{\delta u}^{\prime}\left(\boldsymbol{e}_{s}, \lambda_{s}\right)-b_{\delta u}^{\prime}\left(\boldsymbol{e}_{s}, \lambda_{s}\right)
\end{aligned}
$$

Since the sensitivity of the performance measure needs the sensitivity of the structural bending energy density, it can be extended to the total structural energy density simply using a Boolean operation for all the integral forms. As a result, the sensitivity in Eq. (44) can be rewritten as

$$
\begin{aligned}
\psi^{\prime}=\int_{\Omega} & {\left[g_{, u} \delta \boldsymbol{u}+g_{, e} s\left(h^{-1} \cdot w\left(\boldsymbol{e}_{s}^{\prime}\right)+h^{-1} \cdot w_{\delta \boldsymbol{u}}^{\prime}\left(\boldsymbol{e}_{s}\right)\right)\right.} \\
& +g_{, \nabla e} \cdot \nabla s\left(h^{-1} \cdot w\left(\boldsymbol{e}_{s}^{\prime}\right)+h^{-1} \cdot w_{\delta \boldsymbol{u}}^{\prime}\left(\boldsymbol{e}_{s}\right)\right) \\
& +g_{, \boldsymbol{I}} \cdot \boldsymbol{m}\left(h^{-1} \cdot w\left(\boldsymbol{e}_{s}^{\prime}\right)+h^{-1} \cdot w_{\delta \boldsymbol{u}}^{\prime}\left(\boldsymbol{e}_{s}\right)\right) \\
& \left.+g_{, \nabla \boldsymbol{I}}: \nabla \boldsymbol{m}\left(h^{-1} \cdot w\left(\boldsymbol{e}_{s}^{\prime}\right)+h^{-1} \cdot w_{\delta u}^{\prime}\left(\boldsymbol{e}_{s}\right)\right)\right] d \Omega
\end{aligned}
$$

where the definitions of the integral forms $h(\bullet), w(\bullet), s(\bullet)$ and $\boldsymbol{m}(\bullet)$ are all extended from structural bending energy density to the total structural energy density variables. Substitution of Eq. (48) into Eq. (49) yields

$$
\begin{aligned}
\psi^{\prime}= & \int_{\Omega}\left[g_{, u} \delta \boldsymbol{u}+g_{, e} s\left(h^{-1} \cdot w_{\delta u}^{\prime}\left(\boldsymbol{e}_{s}\right)\right)+g_{, \nabla e} \cdot \nabla s\left(h^{-1} \cdot w_{\delta \boldsymbol{u}}^{\prime}\left(\boldsymbol{e}_{s}\right)\right)\right. \\
& \left.+g_{, \boldsymbol{I}} \cdot \boldsymbol{m}\left(h^{-1} \cdot w_{\delta \boldsymbol{u}}^{\prime}\left(\boldsymbol{e}_{s}\right)\right)+g_{, \nabla \boldsymbol{I}}: \nabla \boldsymbol{m}\left(h^{-1} \cdot w_{\delta \boldsymbol{u}}^{\prime}\left(\boldsymbol{e}_{s}\right)\right)\right] d \Omega \\
& +\ell_{\delta \boldsymbol{u}}^{\prime}\left(\boldsymbol{\lambda}_{s}\right)-a_{\delta \boldsymbol{u}}^{\prime}\left(\boldsymbol{e}_{s}, \boldsymbol{\lambda}_{s}\right)-b_{\delta \boldsymbol{u}}^{\prime}\left(\boldsymbol{e}_{s}, \boldsymbol{\lambda}_{s}\right)
\end{aligned}
$$

In the above equation, the explicitly dependent terms from EFEM such as $\ell_{\delta u}^{\prime}(\bullet), a_{\delta u}^{\prime}(\bullet, \bullet)$ and $b_{\delta u}^{\prime}(\cdot, \bullet)$ have been discussed extensively in Choi and Dong [40,41] for analytical design components, such as structural plates.

Since the conversion integral $w(\bullet)$ is also dependent of the structural design parameters, the calculation of the variation of the conversion integral $w_{\delta u}^{\prime}(\bullet)$ will only involve the structural EFEM model, such that the above sensitivity equation is shown to be an numerical integration variation on the structural model only. Although the adjoint load computation will need the information of the acoustic EBEM model information, the final design sensitivity result is carried on the numerical integration only on the structural part.

\section{Numerical Implementation}

\section{Direct Differentiation Method}

After applying Gaussian quadrature to all integral forms, the matrix equation to solve for the elemental acoustic energy strength is obtained as 


$$
\boldsymbol{\sigma}=\boldsymbol{H}^{-1} \boldsymbol{W} \boldsymbol{E}_{s B}
$$

and the time-averaged acoustic energy density and acoustic energy intensity in the far-field point $\boldsymbol{x}_{0}$ can be obtained using Eqs. (32) and (33).

The variation of the elemental acoustic energy strength can be obtained by the discretization of Eq. (42) as

$$
\boldsymbol{\sigma}^{\prime}=\boldsymbol{H}^{-1} \boldsymbol{W} \boldsymbol{E}_{s B}^{\prime}+\boldsymbol{H}^{-1} \boldsymbol{W}^{\prime} \boldsymbol{E}_{s B}
$$

where the variation of the structural bending energy density vector $\boldsymbol{E}_{s B}^{\prime}$ can be obtained by solving Eq. (39) using a discrete form as

$$
\left[\boldsymbol{K}_{s}+\boldsymbol{J}_{s s}\right]\left\{\boldsymbol{E}_{s}^{\prime}\right\}=\left\{\boldsymbol{F}^{\text {fic }}\right\}
$$

The calculation of the fictitious load vector $\boldsymbol{F}^{\text {fic }}$ can be found in Choi and Dong [40,41] for the parametric design variables. Finally, the variation of the acoustic energy density and acoustic energy intensity can be calculated by discretization of Eqs. (40) and (41) as

$$
\begin{aligned}
& e^{\prime}\left(\boldsymbol{x}_{0}\right)=\boldsymbol{S}^{T} \boldsymbol{\sigma}^{\prime} \\
& \boldsymbol{I}^{\prime}\left(\boldsymbol{x}_{0}\right)=\boldsymbol{M} \boldsymbol{\sigma}^{\prime}
\end{aligned}
$$

Substitution of Eqs. (52)-(55) into Eq. (37) and implementing numerical integration give the sensitivity of the performance measure.

\section{Adjoint Variable Method}

Since the computation of the adjoint load vector for the adjoint variable method depends on the system matrix of both structural EFEM and acoustic EBEM, two separate steps can be used to get the adjoint load vector. First, four separate acoustic adjoint load vectors are defined on an acoustic adjoint re-analysis as

$$
\begin{aligned}
\boldsymbol{H}^{T} \boldsymbol{\xi} & =\boldsymbol{S} \\
\boldsymbol{H}^{T} \boldsymbol{\varsigma}_{i} & =\boldsymbol{M}_{i}, \quad(i=1,2,3)
\end{aligned}
$$

where the acoustic adjoint loads are obtained from the acoustic geometry and the location of the exterior field point $\boldsymbol{x}_{0}$. Vector $\boldsymbol{M}_{i}$ is extracted from matrix $\boldsymbol{M}_{i j}$ with sub-index $i$ fixed. Once the acoustic adjoint responses $\boldsymbol{\xi}$ and $\boldsymbol{\xi}=\left\{\boldsymbol{\zeta}_{1}\right.$, $\left.\varsigma_{2}, \varsigma_{3}\right\}^{T}$ are solved from an acoustic re-analysis, the intermediate adjoint load vectors are defined on the product of the acoustic adjoint responses with the conversion matrix $\boldsymbol{W}$ as

$$
\begin{aligned}
& \boldsymbol{F}_{e}=\boldsymbol{W}^{T} \boldsymbol{\xi} \\
& \boldsymbol{F}_{\boldsymbol{I}}=\boldsymbol{W}^{T} \boldsymbol{\varsigma}
\end{aligned}
$$

where $\boldsymbol{F}_{e}$ is the intermediate adjoint load contributed from the acoustic energy density and $\boldsymbol{F}_{\boldsymbol{I}}$ is the intermediate adjoint load contributed from the acoustic energy intensity at the exterior field point. The substitution of the intermediate adjoint load vectors into the right side of the adjoint equation (45) will give the expression for the structural adjoint load as

$$
\boldsymbol{F}_{a d j}=g_{, e} \boldsymbol{F}_{e}+g_{, \nabla e} \cdot \nabla\left(\boldsymbol{F}_{e}\right)+g_{, \boldsymbol{I}} \cdot\left(\boldsymbol{F}_{\boldsymbol{I}}\right)+g_{, \nabla \boldsymbol{I}}: \nabla\left(\boldsymbol{F}_{\boldsymbol{I}}\right)
$$


With the adjoint variable method, the adjoint response can be approximated by the finite element shape functions

$$
\lambda_{s}=\sum_{i=1}^{N E} N_{s}^{i} \lambda_{s}^{i}=N_{s}^{T} \boldsymbol{\Lambda}_{s}
$$

then the adjoint equation (45) can be eventually discretized as

$$
\left[\boldsymbol{K}_{s}+\boldsymbol{J}_{s s}\right]^{T}\left\{\boldsymbol{\Lambda}_{s}\right\}=\left\{\boldsymbol{F}_{a d j}\right\}
$$

After solving the structural adjoint response $\boldsymbol{\Lambda}_{s}$ at the nodal points, the finite element approximation of the variation of the structural energy bilinear form, structural-structural coupling term and load linear form, the variation of the conversion matrix developed in the previous sections can be applied to derive the variation of the performance measure in the discrete form as

$$
\begin{gathered}
\psi^{\prime}=\iint_{\Omega} g_{, u} \cdot \delta \boldsymbol{u} d \Omega+\boldsymbol{\Lambda}_{s}^{T} \boldsymbol{F}^{f i c}+g_{, e} \boldsymbol{\xi}^{T} \boldsymbol{W}^{\prime} \boldsymbol{E}_{s}+g_{, \nabla e} \cdot \nabla\left(\boldsymbol{\xi}^{T} \boldsymbol{W}^{\prime} \boldsymbol{E}_{s}\right) \\
+g_{, \boldsymbol{I}} \cdot\left(\varsigma^{T} \boldsymbol{W}^{\prime} \boldsymbol{E}_{s}\right)+g_{, \nabla \boldsymbol{I}}: \nabla\left(\varsigma^{T} \boldsymbol{W}^{\prime} \boldsymbol{E}_{s}\right)
\end{gathered}
$$

From the definition of the acoustic adjoint load vectors in Eqs. (56) and (57), it is shown that as long as the geometry of the boundary element model holds fixed, the acoustic adjoint load vectors, thus the acoustic adjoint responses remain unchanged. This provides great advantages for the parametric design optimization of the structural-acoustic model, since as long as the structural geometry does not change, the parametric design variables such as panel thickness and material damping will not influence the computation of the acoustic loads and acoustic adjoint responses. However, the structural adjoint loads are denpendent on structural designs through the conversion matrix $\boldsymbol{W}$. As a result, Eqs. (58)-(60) need to be evaluated at each design iteration in a sizing design optimization process.

\section{Numerical Examples}

\section{Design Sensitivity Analysis of Radiation of Simplified Van Model In Air}

Radiated noise from vibrating structure to the exterior domain is as important as the interior noise since it generates noise pollution. The exterior noise radiated from vibrating vehicle structure is one of the most common radiation problems to study. To design vehicle structure such that the radiated noise level is controlled under regulatory limit is one of the most important tasks for NVH design. A practical application of EFEM-EBEM is to design a vehicle structure with optimized NVH performance and ride quality at high frequency. For this purpose, a simplified passenger vehicle model is constructed and studied (Fig. 2). The corresponding EFEM model (Fig. 3) is comprised of 118 structural plate elements, which are integrated through 56 structural-structural joints. An analytical method developed by Langley and Heron [21] is used to compute the power transfer coefficients between plate members and the radiation efficiency method is used to compute the power transfer coefficients between plate members and acoustic domain.

The passenger vehicle model is comprised of seven different structural panels, made of aluminum. Those panels have the property of Young's modulus $E=71 \mathrm{Gpa}$, Poisson's ratio $v=0.33$, mass density $\rho_{s}=2700 \mathrm{~kg} / \mathrm{m}^{3}$, and all the panels have the same thickness $h=10 \mathrm{~mm}$, the hysteresis damping factor of $\eta=0.01$ is used for each panel. The noise is radiated into acoustic space filled with air, of mass density $\rho_{0}=1.02 \mathrm{~kg} / \mathrm{m}^{3}$, hysteresis damping $\eta_{a}=0.001$ and wave speed $c_{0}=343 \mathrm{~m} / \mathrm{s}$.

A structural EFEM analysis is performed first without considering the interior acoustic domain to obtain the structural energy density distribution. Since the structural model does not have any inner part, the acoustic energy boundary element model is the same as the structural finite element model in Fig. 3. A recovery point $P_{0}$ is chosen to be located in front of the vehicle with a coordinate at $(8.0,-0.5,0.5)$ as shown in Fig. 2. An acoustic EBEM analysis following the structural EFEM analysis predicts the noise level at the recovery point is $70.1 \mathrm{~dB}$ in air. The proposed adjoint design sensitivity method is carried out to study the sensitivity effect of structural designs on the radiated noise. Thickness and damping of each structural panel are selected as design variables and $1 \%$ of the initial design is perturbed where central finite difference method is used to verify the sensitivity coefficients. The comparisons of the results from the proposed design sensitivity method and central finite difference method are shown in Table 1 for 
thickness and damping design variable respectively. An excellent agreement is observed between these two sets of numerical results, which prove the accuracy of the proposed design sensitivity method.

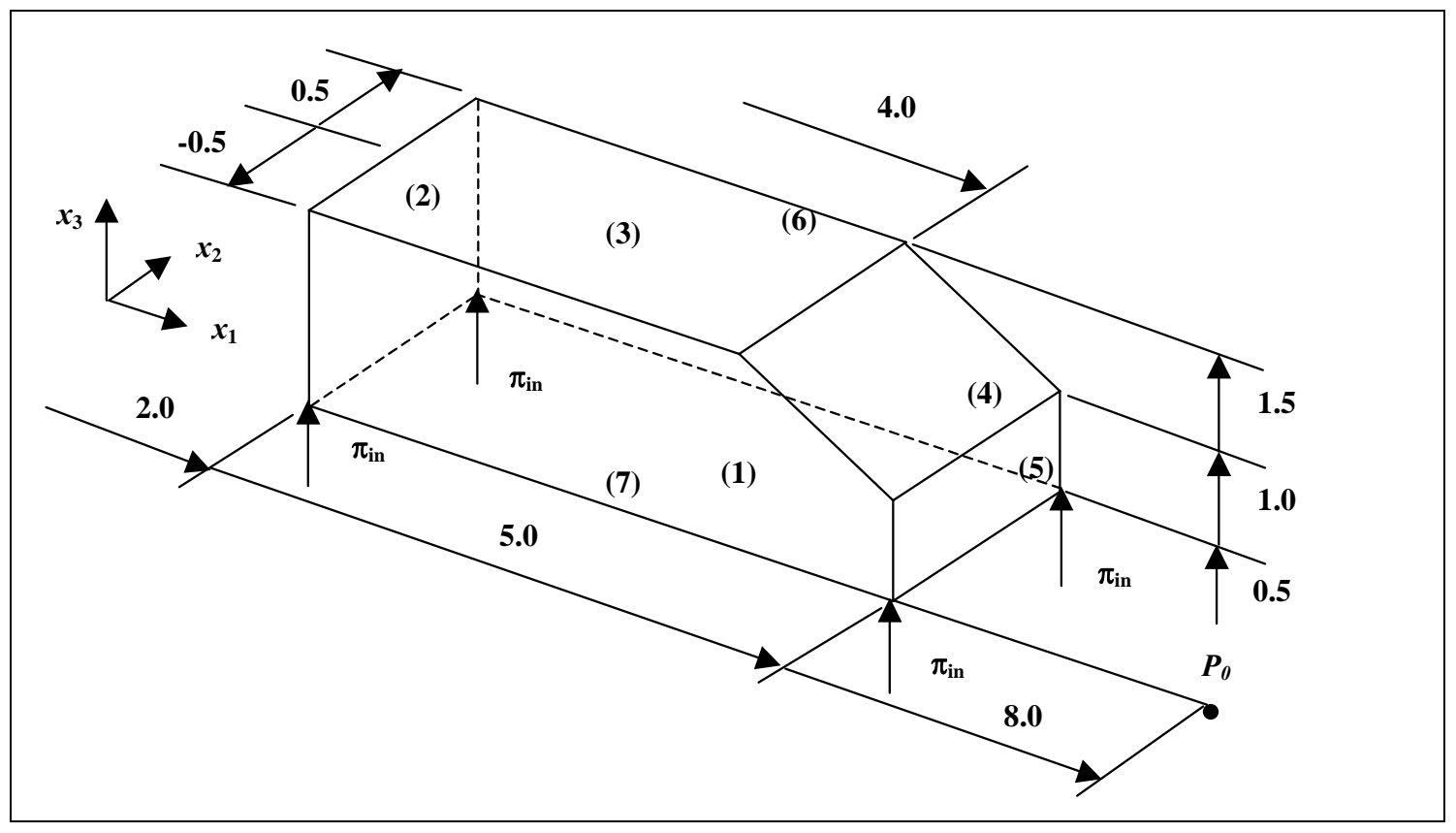

Figure 2 Recovery Point in Exterior Domain of Simplified Van Model

The sensitivity information shown in Table1 presents the different effects of the structural designs on the noise level at the recovery point $P_{0}$. All the damping designs have negative contributions to the radiated noise, and most of the thickness designs also have negative contributions, except panel 2 and 3 , which have positive sensitivity coefficients, which means, the increase of the thickness in panel 2 and 3 will not help reduce the radiated noise at $P_{0}$, but instead increase it.

To better understand the sensitivity results, the sensitivity coefficients of the noise level at the recovery point $P_{0}$ with respect to the element thickness and damping factors are calculated and the results are plotted in Fig. 4 and Fig. 5. It clearly explains that the structural damping always has negative effect on the noise radiation, no matter interior or exterior noise. Increasing structural damping will always help noise reduction. Compared with the damping design, contribution from the thickness design to the noise radiation is significantly different considering the location of the recovery point and the role of each structural panel in the total energy flow in the structure. 
Table 1 Design Sensitivity Analysis Results for Exterior Noise Radiation from Simplified Van Model

\begin{tabular}{|c|c|c|c|c|c|c|}
\hline \multicolumn{2}{|c|}{ Design Variables } & \multicolumn{2}{|c|}{ Performance Measure } & \multirow{2}{*}{$\begin{array}{l}\text { FDM } \\
\delta \psi / \delta u\end{array}$} & \multirow{2}{*}{$\begin{array}{c}\text { AVM } \\
\psi^{\prime}\end{array}$} & \multirow{2}{*}{$\begin{array}{c}\text { Ratio } \\
(\%)\end{array}$} \\
\hline & & $\psi(u-\delta u)$ & $\psi(u+\delta u)$ & & & \\
\hline \multirow{2}{*}{$\begin{array}{c}\text { Panel } \\
1\end{array}$} & Thickness $h_{1}$ & 70.212718 & 70.005966 & $-0.103376 \mathrm{E}+04$ & $-0.103371 \mathrm{E}+04$ & 100.01 \\
\hline & Damping $\eta_{1}$ & 70.122588 & 70.108509 & $-0.703957 \mathrm{E}+03$ & $-0.703956 \mathrm{E}+03$ & 100.00 \\
\hline \multirow{2}{*}{$\begin{array}{c}\text { Panel } \\
2\end{array}$} & Thickness $h_{2}$ & 70.116585 & 70.118256 & $0.835712 \mathrm{E}+01$ & $0.835866 \mathrm{E}+01$ & 99.98 \\
\hline & Damping $\eta_{2}$ & 70.117710 & 70.113976 & $-0.186710 \mathrm{E}+03$ & $-0.186710 \mathrm{E}+03$ & 100.00 \\
\hline \multirow{2}{*}{$\begin{array}{c}\text { Panel } \\
3\end{array}$} & Thickness $h_{3}$ & 70.116369 & 70.117665 & $0.648013 \mathrm{E}+01$ & $0.648394 \mathrm{E}+01$ & 99.94 \\
\hline & Damping $\eta_{3}$ & 70.119146 & 70.111944 & $-0.360090 \mathrm{E}+03$ & $-0.360090 \mathrm{E}+03$ & 100.00 \\
\hline \multirow{2}{*}{$\begin{array}{c}\text { Panel } \\
4\end{array}$} & Thickness $h_{4}$ & 70.119748 & 70.109103 & $-0.532236 \mathrm{E}+02$ & $-0.532125 \mathrm{E}+02$ & 100.02 \\
\hline & Damping $\eta_{4}$ & 70.117610 & 70.113476 & $-0.206683 \mathrm{E}+03$ & $-0.206683 \mathrm{E}+03$ & 100.00 \\
\hline \multirow{2}{*}{$\begin{array}{l}\text { Panel } \\
5\end{array}$} & Thickness $h_{5}$ & 70.117944 & 70.110138 & $-0.390287 \mathrm{E}+02$ & $-0.390208 \mathrm{E}+02$ & 100.02 \\
\hline & Damping $\eta_{5}$ & 70.116552 & 70.114534 & $-0.100905 \mathrm{E}+03$ & $-0.100905 \mathrm{E}+03$ & 100.00 \\
\hline \multirow{2}{*}{$\begin{array}{c}\text { Panel } \\
6\end{array}$} & Thickness $h_{6}$ & 70.119946 & 70.114788 & $-0.257887 \mathrm{E}+02$ & $-0.257789 \mathrm{E}+02$ & 100.04 \\
\hline & Damping $\eta_{6}$ & 70.120740 & 70.110355 & $-0.519246 \mathrm{E}+03$ & $-0.519245 \mathrm{E}+03$ & 100.00 \\
\hline \multirow{2}{*}{$\begin{array}{c}\text { Panel } \\
7\end{array}$} & Thickness $h_{7}$ & 70.121715 & 70.104112 & $-0.880149 \mathrm{E}+02$ & $-0.880005 \mathrm{E}+02$ & 100.02 \\
\hline & Damping $\eta_{7}$ & 70.120835 & 70.110259 & $-0.528774 \mathrm{E}+03$ & $-0.528774 \mathrm{E}+03$ & 100.00 \\
\hline
\end{tabular}

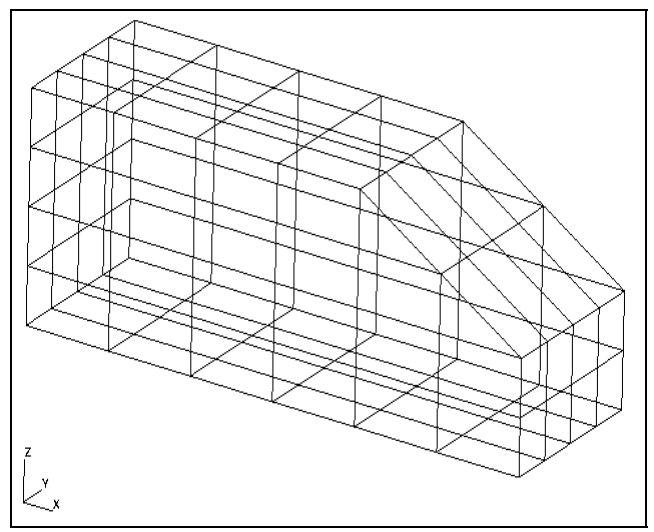

Figure 3 Energy Finite Element Model of Simplified Van Model 


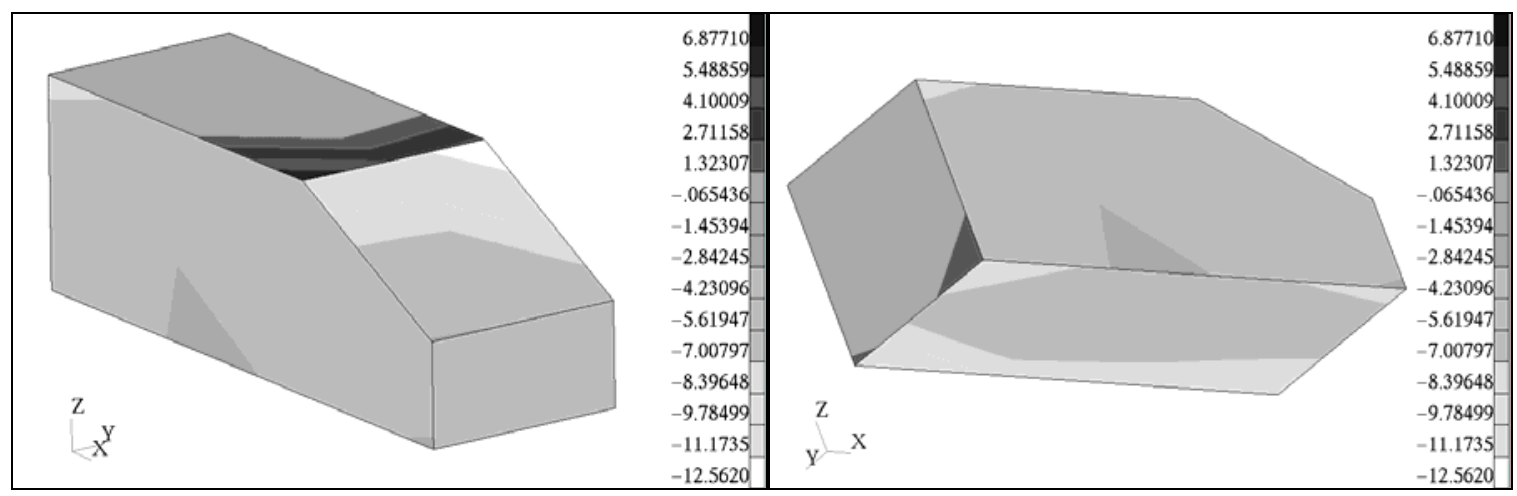

a) View 1

b) View 2

Figure 4 Design Sensitivity Plot of Radiated Noise With Respect to Element Thickness Design for Simplified Van Model

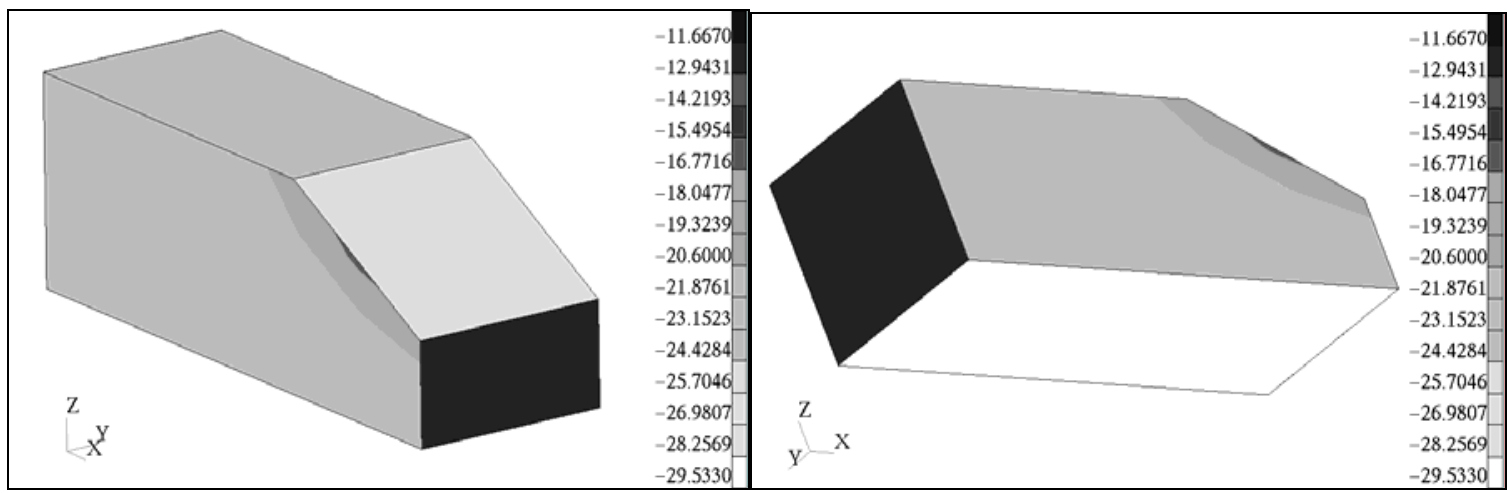
a) View 1
b) View 2

Figure 5 Design Sensitivity Plot of Radiated Noise With Repsect to Element Damping Design for Simplified Van Model

\section{Design Sensitivity Analysis of Underwater Radiation of Undersea Vehicle Model}

High frequency noise radiation also plays an important role in naval structures as in automotive and aerospace structures. For example, how to reduce the noise radiated from a vibrating undersea ship to make it avoid being traced and identified is an attractive topic in naval structure design. To this end, a small undersea vehicle model is constructed and the noise radiation in water is analyzed. The vehicle model is $6.0 \mathrm{~m}$ long, and the maximum inner diameter is $0.522 \mathrm{~m}$. The thickness of the vehicle skin is $10 \mathrm{~mm}$, and all of the 4 bulkheads have the same thickness 
of $25.4 \mathrm{~mm}$. The energy finite element model (Fig. 6) is comprised of 1744 nodes and 1060 elements. There are totally 590 structural joints in the model. The structural parts have the material properties with Young's modulus $E$ $=70 \mathrm{GPa}$, Poisson's ratio $v=0.3$, mass density $\rho=2,700 \mathrm{~kg} / \mathrm{m}^{3}$, and hysteresis damping factor $\eta=0.01$. Water is the fluid with which the undersea model interacts, which has mass density $\rho_{0}=1,000 \mathrm{~kg} / \mathrm{m}^{3}$, wave speed $c_{0}=1,500$ $\mathrm{m} / \mathrm{s}$ and hysteresis damping factor $\eta=0.001$.

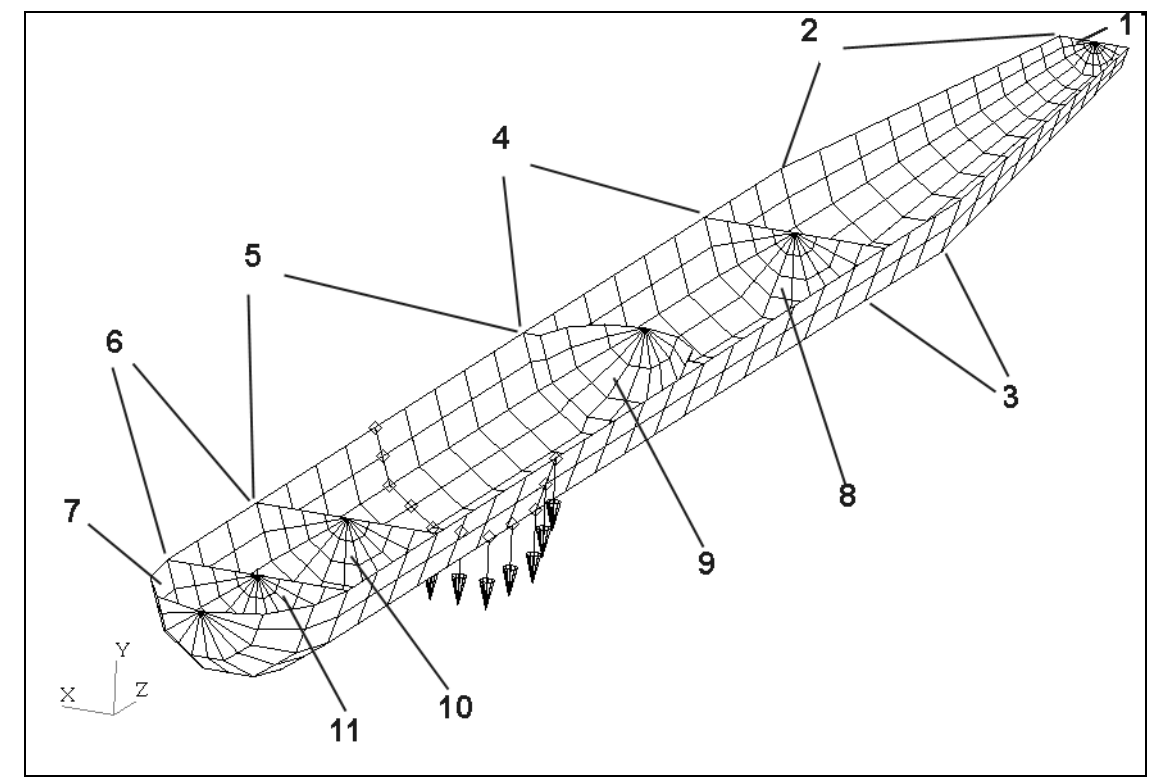

Figure 6 Energy Finite Element Model of Small Undersea Vehicle Model

Because of the differences of the acoustic wave numbers and the effective structural mass density of the structural plate in light fluid (air) and dense fluid (water), the radiation damping, which represents the capacity of the structural plates to radiate acoustic energy, will be different in dense fluid compared to that in light fluid. For example, for an aluminum plate with the dimension of $1 \mathrm{~m} \times 1 \mathrm{~m} \times 0.001 \mathrm{~m}$, its radiation damping in both air and water can be computed using Eq. (25) and the Leppington method (Eq. 27) for radiation efficiency in the frequency range between $100 \mathrm{~Hz}$ to $10000 \mathrm{~Hz}$ and compared in Fig. 7. Since the radiation damping is included in the governing equation of the structural bending power flow [22], the difference in the radiation damping could have large effect on the governing equation of the structural vibration. For example, the radiation damping of the aluminum plate is ranging from $0.002 \sim 0.0025$ in water, which is about $20 \sim 25 \%$ of its original hysteresis damping of the bending motion. However, the radiation damping in air is less than 0.00025 , which is not comparable to the original damping of structural bending motion, and its contribution will be much smaller than that in water. 


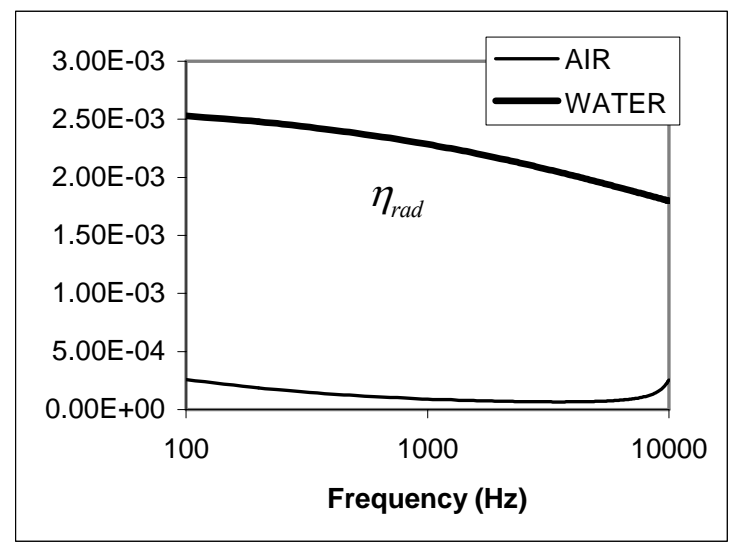

Figure 7 Radiation Damping of Aluminum Plate in Air and Water

In addition to the radiation damping, the existence of the dense fluid could affect the power transfer coefficients between the structural panels significantly. The effective structural mass density $\rho_{s}$, the bending wave number $k_{s B}$ and the bending group speed $c_{g B}$ for the structural plates in dense fluid, which are characterized by parameter $\alpha$ in Eq. (26), are included in the analytical method proposed by Langley and Heron [21] to compute power transfer coefficients for structural plates in dense fluid [22]. Figure 8 compares the power transfer coefficients between two identical plates with the same material properties $\left(E=209 \mathrm{GPa}, v=0.3, \rho=7,800 \mathrm{~kg} / \mathrm{m}^{3}\right)$ in vacuum, light fluid (air) and dense fluid (water). The two plates have the same dimension of $1 \mathrm{~m} \times 1 \mathrm{~m} \times 0.001 \mathrm{~m}$ and the power transfer coefficients are computed and compared for different junction angle from $0^{\circ}$ to $180^{\circ}$. In Fig. $8, \tau_{12}^{B B}$ represents the the ratio of energy transfer from the bending energy in plate 1 to the bending energy in plate 2, and $\tau_{11}^{B B}$ stand for the ration of energy trasnfer from the bending energy in plate 1 to the bending energy in plate 1 itself. It is shown that the light fluid (air) does not cause any difference in the power transfer properties compared to those in vacuum. On the contrary, the effect of the dense fluid (water) on the power transfer coefficients is clear, since the power transfer coefficients around $10^{\circ}$ to $30^{\circ}$ and $140^{\circ}$ to $170^{\circ}$ are significantly different in water compared with those in vacuum and air. It emphasizes the contribution of fluid effect on high frequency power flow between structural components. 


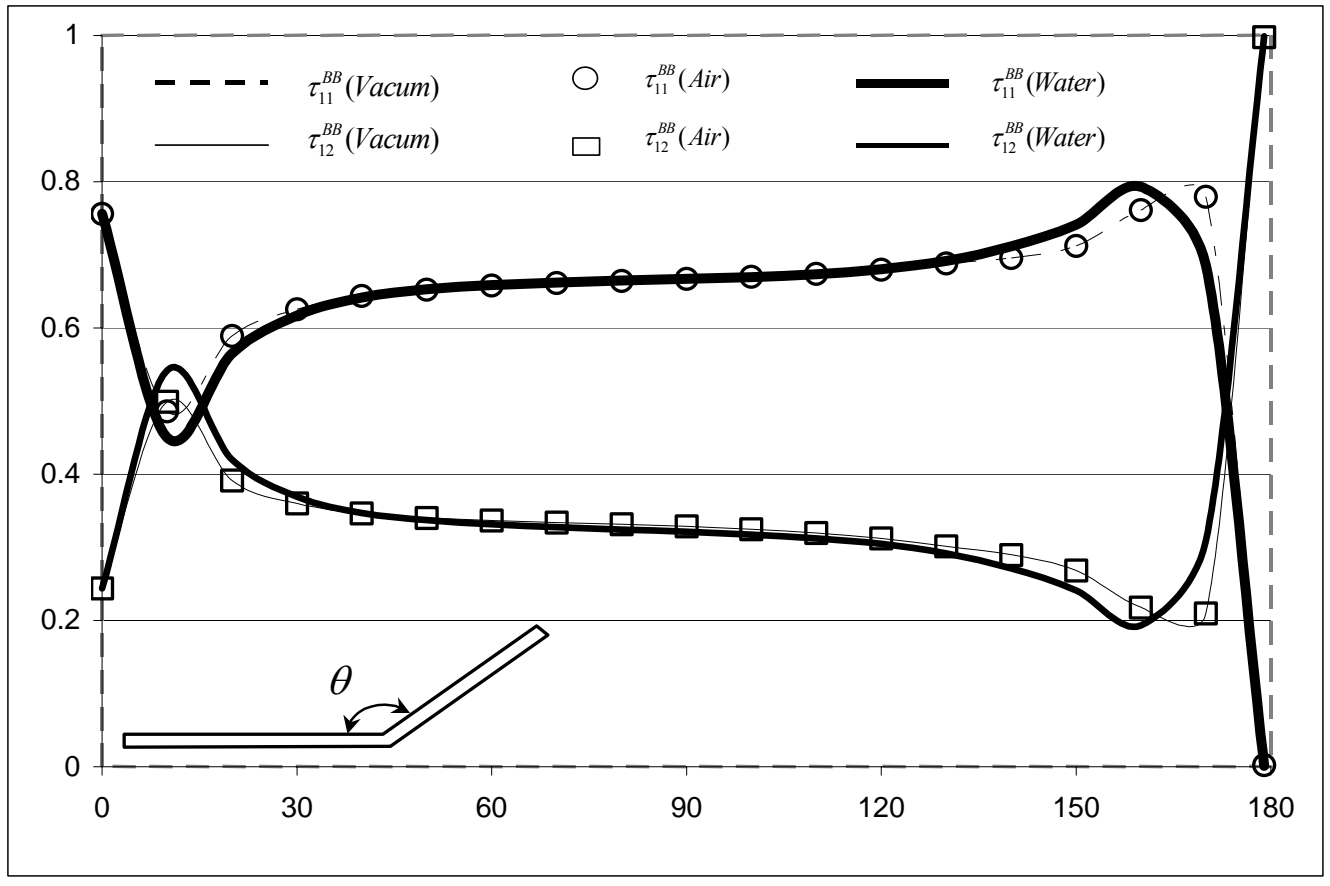

Figure 8 Power Transfer Coefficients of Two Angled Plates in Vacuum, Air and Water

The undersea vehicle model can be divided into 11 substructures as shown in Fig. 6. Axi-symmetric excitation is applied in the middle of substructure 5. The structural energy distribution at frequency $f=2000 \mathrm{~Hz}$ is plotted in Fig. 9, from which we can see that most of the energy gather around substructure 5, and the 4 bulkheads can be used to isolate the energy flow between the substructures. 


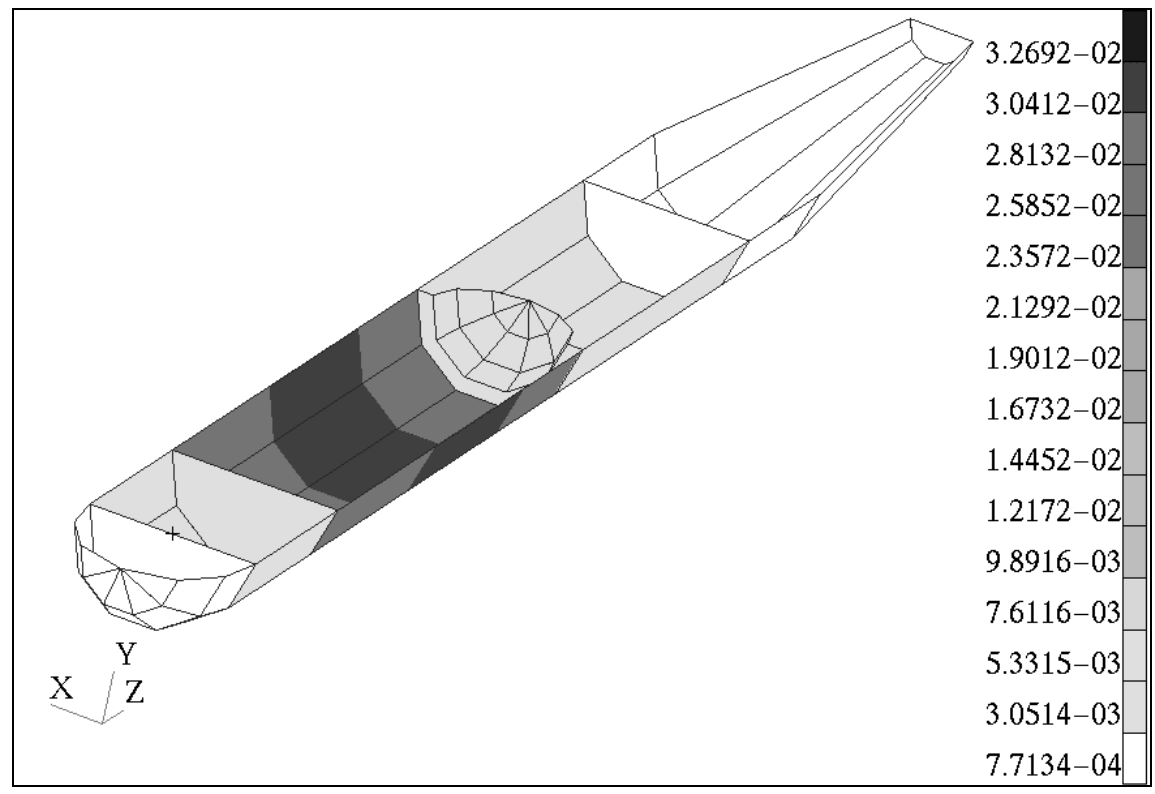

Figure 9 Structural Energy Density Distribution of Small Undersea Vehicle Model

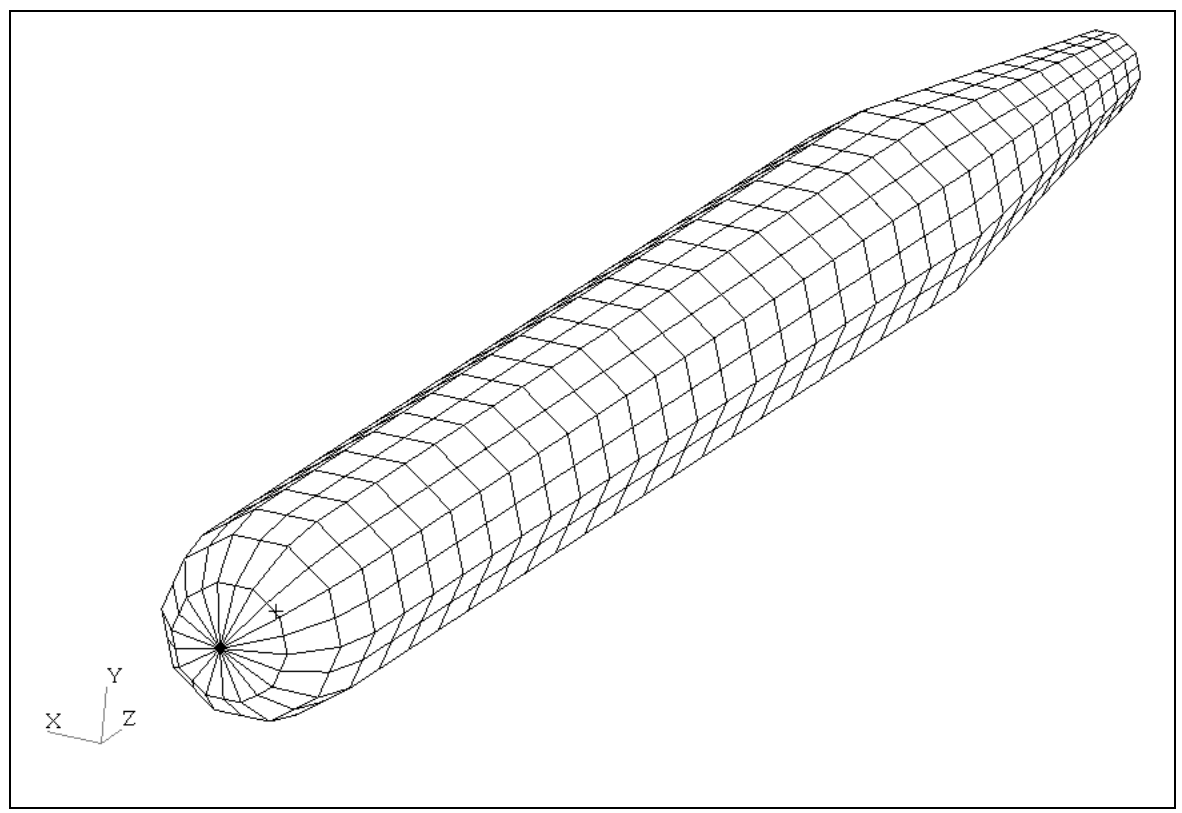

Figure 10 Energy Boundary Element Model of Small Undersea Vehicle Model 


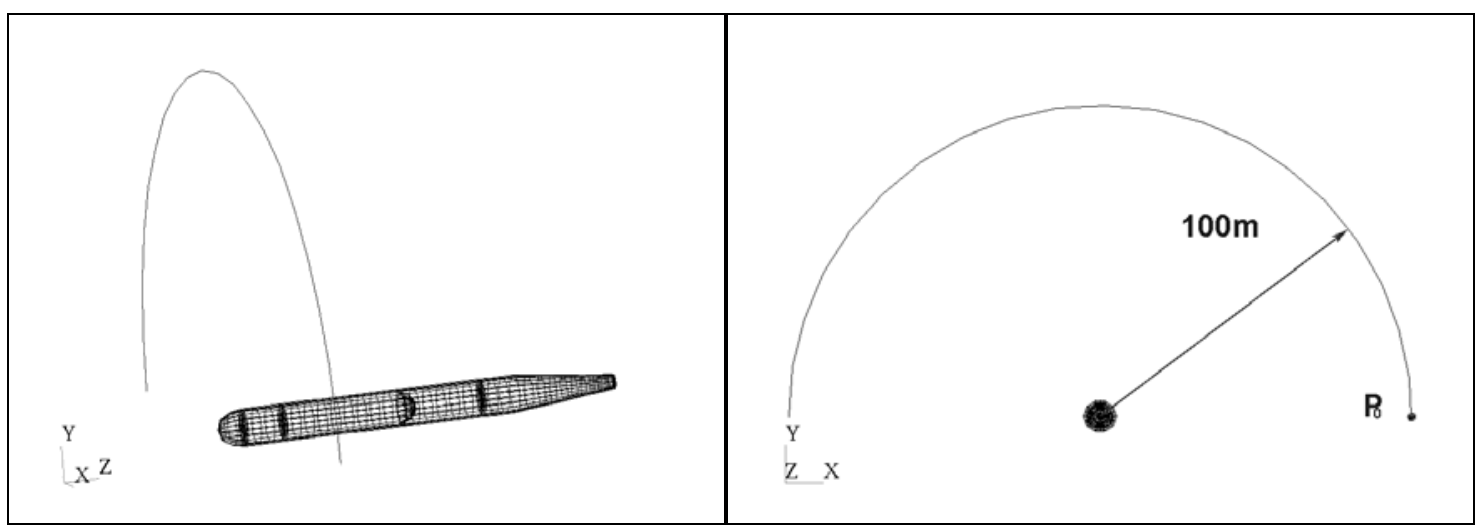

a) View 1

b) View 2

Figure 11 Exterior Noise Recovery Position of Small Undersea Vehicle Model

While the energy finite element model consists both inner parts and outer parts, the energy boundary element model only consists the outer parts of the structural model (Fig. 10). The structural energy density is converted to the power flow on each structural element, which is then used as the boundary condition to solve for the acoustic energy strength for each boundary element. At the end, the radiated noise at an arbitrary exterior point can be computed by using Green's function. To predict the exterior radiation noise by the vibrating vehicle model, a series of points with distance $100 \mathrm{~m}$ from the center of the vehicle model is selected as recovery points as shown in Fig. 11. Since the model and excitation are axi-symmetric, all the points on the circle in Fig. 11 will have the same noise level. Therefore, the noise level on the entire circle can be represented by only one point, for example, point $P_{0}$. Furthermore, if each substructure of the vehicle model can be considered as a design component, the design sensitivity coefficients of the noise level on the entire circle will also be equivalent to the design sensitivity coefficients of the noise level on point $P_{0}$. As a result of the radiation analysis using EFEM-EBEM, the noise level at the circle is predicted at $118.87 \mathrm{~dB}$ when $f=2000 \mathrm{~Hz}$. Following the design sensitivity analysis procedure discussed above, the sensitivity coefficients of the noise level with respect to the thickness and damping factor of each substructure are calculated and summarized in Table 2. Without exception, all the damping factors have negative sensitivity coefficients. For the thickness design, thicknesses of substructure 1 through 7 have positive coefficients. Among the 4 bulkheads, substructure 9 has positive sensitivity coefficients while all the other three have negative sensitivity coefficients. It shows that at the current design, increase damping of each structural panel and increase thickness of substructure 1 through 7 and 9 , while decrease thickness of other substructures is the appropriate way to reduce the noise radiated at the points of interest. 
Table 2 Design Sensitivity Analysis Results for Exterior Noise Radiation from Small Undersea Vehicle Model

\begin{tabular}{|c|c|c|c|c|c|c|}
\hline \multicolumn{2}{|c|}{ Design Variables } & \multicolumn{2}{|c|}{ Performance Measure } & \multirow{2}{*}{$\begin{array}{l}\text { FDM } \\
\delta \psi / \delta u\end{array}$} & \multirow{2}{*}{$\begin{array}{c}\text { AVM } \\
\psi^{\prime}\end{array}$} & \multirow{2}{*}{$\begin{array}{c}\text { Ratio } \\
(\%)\end{array}$} \\
\hline & & $\psi(u-\delta u)$ & $\psi(u+\delta u)$ & & & \\
\hline \multirow{2}{*}{$\begin{array}{c}\text { Substructure } \\
1\end{array}$} & Thickness $h_{1}$ & 118.872284 & 118.872290 & $0.325359 \mathrm{E}-01$ & $0.325213 \tilde{\mathrm{E}} 01$ & 100.04 \\
\hline & Damping $\eta_{1}$ & 118.872286 & 118.872274 & $-0.594010 \tilde{\mathrm{E}} 01$ & $-0.594010 \tilde{\mathrm{E}} 01$ & 100.00 \\
\hline \multirow{2}{*}{$\begin{array}{c}\text { Substructure } \\
2\end{array}$} & Thickness $h_{2}$ & 118.871904 & 118.872498 & $0.296974 \mathrm{E}+01$ & $0.296954 \mathrm{E}+01$ & 100.01 \\
\hline & Damping $\eta_{2}$ & 118.872627 & 118.871934 & $-0.346370 \mathrm{E}+01$ & $-0.346369 \mathrm{E}+01$ & 100.00 \\
\hline \multirow{2}{*}{$\begin{array}{c}\text { Substructure } \\
3\end{array}$} & Thickness $h_{3}$ & 118.872107 & 118.872469 & $0.181275 \mathrm{E}+01$ & $0.181259 \mathrm{E}+01$ & 100.00 \\
\hline & Damping $\eta_{3}$ & 118.872448 & 118.872112 & $-0.168031 \mathrm{E}+01$ & $-0.168031 \mathrm{E}+01$ & 100.00 \\
\hline \multirow{2}{*}{$\begin{array}{c}\text { Substructure } \\
4\end{array}$} & Thickness $h_{4}$ & 118.871137 & 118.873288 & $0.107525 \mathrm{E}+02$ & $0.107523 \mathrm{E}+02$ & 100.00 \\
\hline & Damping $\eta_{4}$ & 118.873321 & 118.871244 & $-0.103865 \mathrm{E}+02$ & $-0.103864 \mathrm{E}+02$ & 100.00 \\
\hline \multirow{2}{*}{$\begin{array}{c}\text { Substructure } \\
5\end{array}$} & Thickness $h_{5}$ & 118.862150 & 118.882652 & $0.102510 \mathrm{E}+03$ & $0.102501 \mathrm{E}+03$ & 100.00 \\
\hline & Damping $\eta_{5}$ & 118.884215 & 118.860378 & $-0.119186 \mathrm{E}+03$ & $-0.119185 E+03$ & 100.00 \\
\hline \multirow{2}{*}{$\begin{array}{c}\text { Substructure } \\
6\end{array}$} & Thickness $h_{6}$ & 118.871270 & 118.872900 & $0.815108 \mathrm{E}+01$ & $0.815076 \mathrm{E}+01$ & 100.00 \\
\hline & Damping $\eta_{6}$ & 118.873007 & 118.871556 & $-0.725600 \mathrm{E}+01$ & $-0.725598 \mathrm{E}+01$ & 100.00 \\
\hline \multirow{2}{*}{$\begin{array}{c}\text { Substructure } \\
7\end{array}$} & Thickness $h_{7}$ & 118.872034 & 118.872495 & $0.230714 \mathrm{E}+01$ & $0.230695 \mathrm{E}+01$ & 100.00 \\
\hline & Damping $\eta_{7}$ & 118.872536 & 118.872025 & $-0.255530 \mathrm{E}+01$ & $-0.255529 \mathrm{E}+01$ & 100.00 \\
\hline \multirow{2}{*}{$\begin{array}{c}\text { Substructure } \\
8\end{array}$} & Thickness $h_{8}$ & 118.872356 & 118.872233 & $-0.242408 \mathrm{E}+00$ & $-0.242408 \mathrm{E}+00$ & 100.00 \\
\hline & Damping $\eta_{8}$ & 118.872303 & 118.872258 & $-0.220730 \mathrm{E}+00$ & $-0.220730 \mathrm{E}+00$ & 100.00 \\
\hline \multirow{2}{*}{$\begin{array}{c}\text { Substructure } \\
9\end{array}$} & Thickness $h_{9}$ & 118.872161 & 118.872377 & $0.424456 \mathrm{E}+00$ & $0.424435 \mathrm{E}+00$ & 100.00 \\
\hline & Damping $\eta_{9}$ & 118.872430 & 118.872130 & $-0.149853 \mathrm{E}+01$ & $-0.149853 \mathrm{E}+01$ & 100.00 \\
\hline \multirow{2}{*}{$\begin{array}{c}\text { Substructure } \\
10\end{array}$} & Thickness $h_{10}$ & 118.872409 & 118.872148 & $-0.513201 \mathrm{E}+00$ & $-0.513172 \mathrm{E}+00$ & 100.00 \\
\hline & Damping $\eta_{10}$ & 118.872385 & 118.872175 & $-0.104580 \mathrm{E}+01$ & $-0.104580 \mathrm{E}+01$ & 100.00 \\
\hline \multirow{2}{*}{$\begin{array}{c}\text { Substructure } \\
11\end{array}$} & Thickness $h_{11}$ & 118.872288 & 118.872193 & $-0.188094 \mathrm{E}+00$ & $-0.188102 \mathrm{E}+00$ & 100.00 \\
\hline & Damping $\eta_{11}$ & 118.872319 & 118.872241 & $-0.387373 E+00$ & $-0.387372 \mathrm{E}+00$ & 100.00 \\
\hline
\end{tabular}

Figures 12 and 13 plot the design sensitivity coefficients of the exterior noise level with respect to the element thickness and damping design variables respectively. In Fig. 12, black color stands for maximum positive sensitivity coefficients, while white color stands for negative sensitivity coefficients. Substructure 5 has the highest positive sensitivity coefficients, and substructures $8,10,11$ have negative sensitivity coefficients. The results are consistent with the numerical results in Table 2. In Fig. 13, all the sensitivity coefficients are negative, with black color stands for larger sensitivity value, white color stands for smaller sensitivity value. It is shown that the substructure 5 has the highest negative contribution to the exterior noise level, which is followed by the other substructures and bulkheads. With the graphic information in Figs. 12 and 13 along with the tabular results in Table 2, a design engineer will be able to know how to perturb the initial design to achieve a better and eventually an optimum design for best NVH performance with least usage of material. 


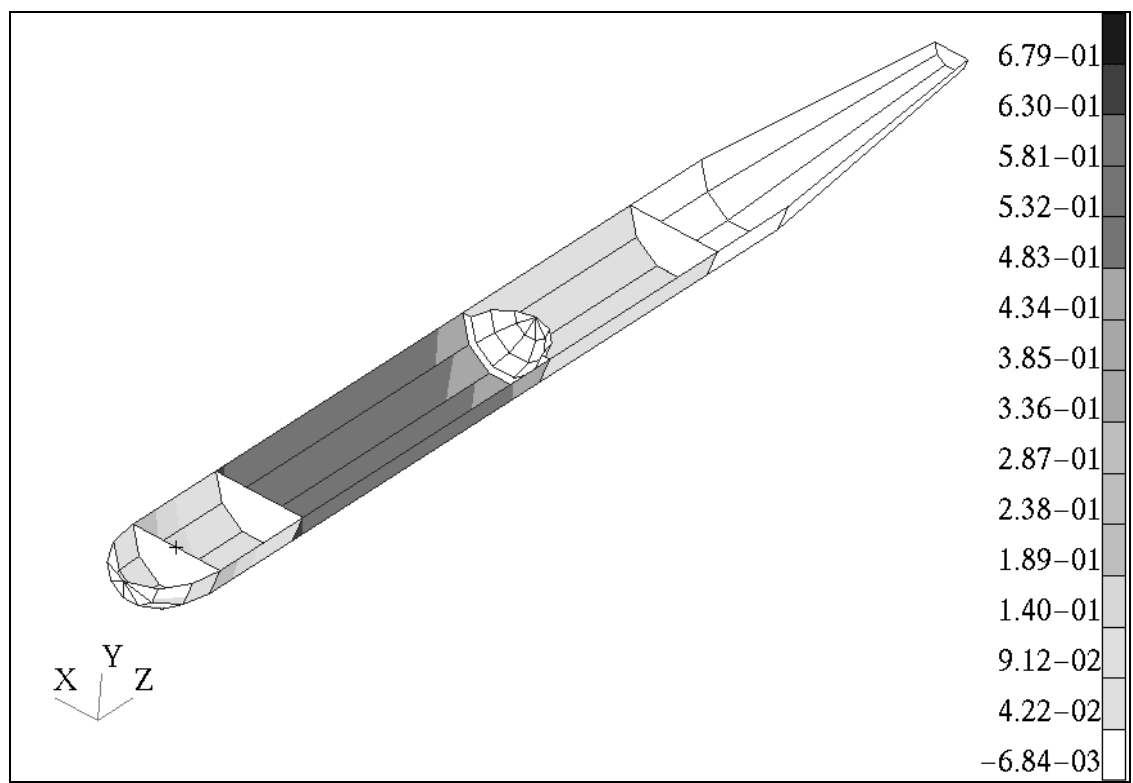

Figure 12 Design Sensitivity Plot of Radiated Noise With Respect to Element Thickness Design for Undersea Vehicle Model

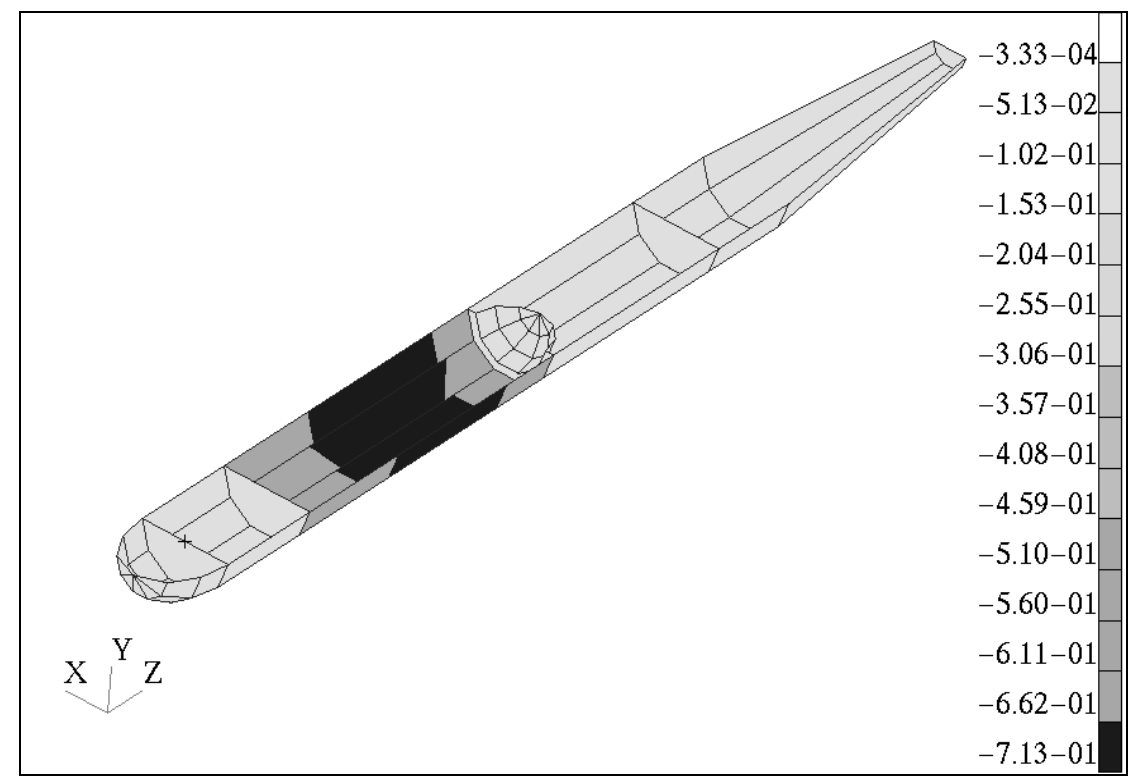

Figure 13 Design Sensitivity Plot of Radiated Noise Versus Element Damping Design for Undersea Vehicle Model 


\section{Multi-Objective Design Optimization of Underwater Noise Reduction}

In this section, a design optimization problem is formulated and solved for the undersea vehicle model. The DSA formulation derived in the previous sections will be integrated into the design optimization program to carry out design optimization process.

The design optimization process is illustrated in Fig. 14. The EFEM is used to compute the structural energy distribution and power radiated from the structural plate, which will be used as boundary conditions for EBEM to predict the noise radiation at far-field points. Using the sequential adjoint variable method, the DSA calculates the derivative of the far-field radiation noise with respect to the design variables, and all the information are utilized by the design optimization algorithm (such as SLP and SQP, etc) to search for the optimum design.

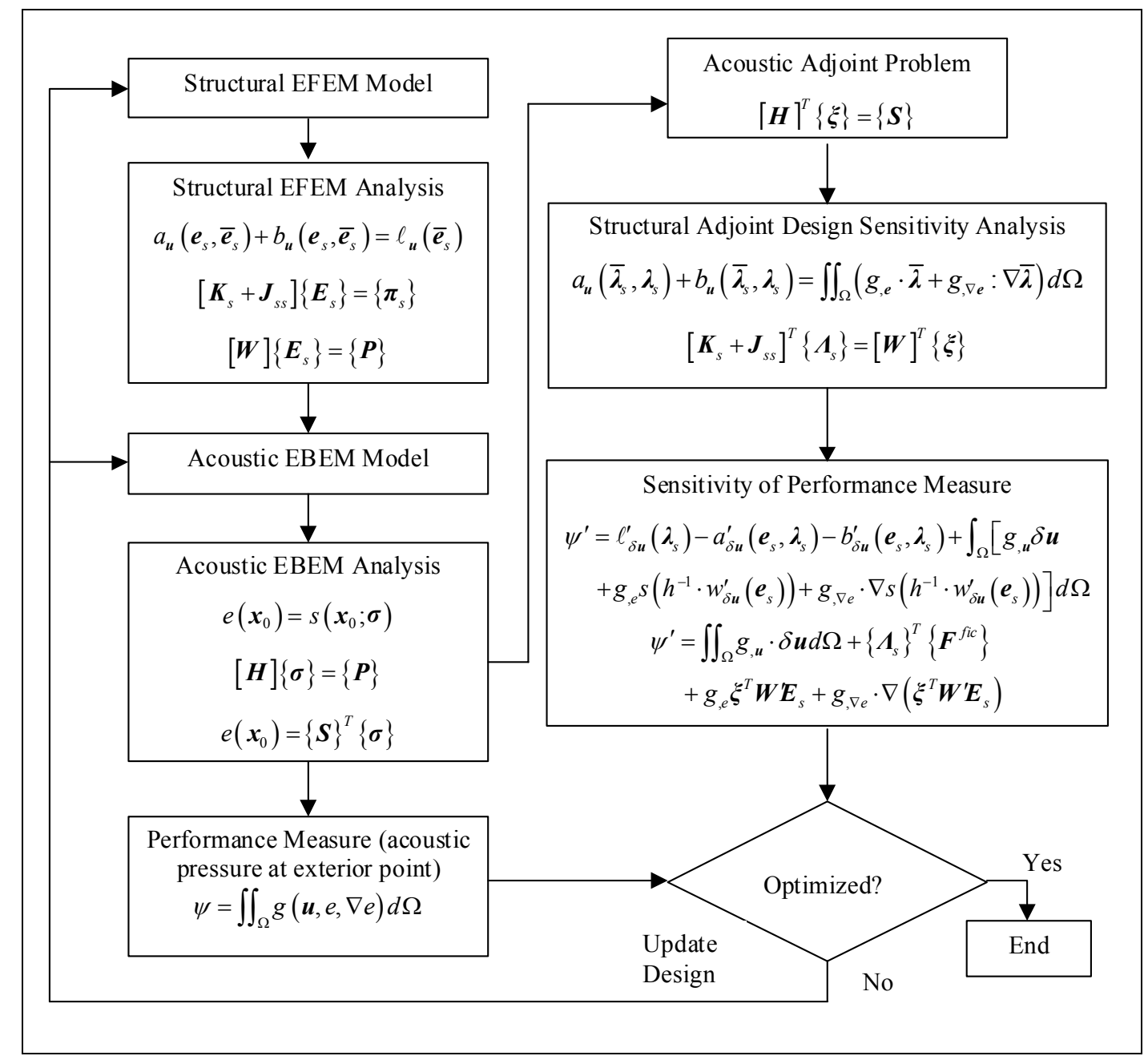

Figure 14 Design Optimization Procedure for High Frequency Radiation Problem Using Energy Finite Element Method and Energy Boundary Element Method 
The purpose of the design optimization is to reduce the noise level at far-field points at a high frequency range from 1600 to $2500 \mathrm{~Hz}$ with the minimum vehicle mass. The first performance measure will be selected as the farfield noise level at point $P_{0}$. Meanwhile, since the potential optimum design could cause the increase in energy density distribution on the structural model, which on the other hand, explains the increase in structural stress level. To avoid the structural energy density increase, another performance measure should be selected to represent the highest energy density on the structural model. The computational results show that the highest energy density occurs at Node 752 of the structural energy finite element model, which will be used as the structural performance measure. As a result, the acoustic performance measure at $\mathrm{P}_{0}$ and the structural performance measure at Node 752 are obtained at the center frequencies of the $1 / 3$ octave bands in the range from 1600 to $2500 \mathrm{~Hz}$ and are listed in the Table 3.

Table 3 Performance Measures at Initial design

\begin{tabular}{ccc}
\hline $\begin{array}{c}\text { Frequency } \\
(\mathrm{Hz})\end{array}$ & $\begin{array}{c}\text { Acoustic Pressure } \\
(\mathrm{dB}, \mathrm{RE} 2.0 \mathrm{E}-5)\end{array}$ & $\begin{array}{c}\text { Structural Energy } \\
\text { Density } \\
(\mathrm{dB}, \text { RE1.0E-12) }\end{array}$ \\
\hline 1600 & 118.92 & 126.02 \\
\hline 1692 & 118.91 & 125.84 \\
\hline 1789 & 118.90 & 125.58 \\
\hline 1891 & 118.89 & 125.37 \\
\hline 2000 & 118.87 & 125.17 \\
\hline 2115 & 118.86 & 125.06 \\
\hline 2236 & 118.84 & 124.79 \\
\hline 2364 & 118.83 & 124.52 \\
\hline 2500 & 118.82 & 124.32 \\
\hline
\end{tabular}

Since the mass cost will serve as design objective, the above noise level and structural energy density will serve as design constraints. The maximum noise level occurs at $f=1600 \mathrm{~Hz}$, which is $118.9 \mathrm{~dB}$ with reference value of 2.0E-5 Pa, so one of the constraints is to reduce the highest pressure level to $116.00 \mathrm{~dB}$ with reference value of $1.0 \mathrm{E}-$ $12 \mathrm{~J} / \mathrm{m}^{2}$, which means $2.9 \mathrm{~dB}$ reduction, equivalent to $28.4 \%$ noise reduction. At the same time, the highest structural energy density occurs at $f=1600 \mathrm{~Hz}$, which is $126.0 \mathrm{~dB}$, the design optimization needs to keep the highest structural energy density level under $126.0 \mathrm{~dB}$. As a result, the design constraints are to reduce the noise level at $\mathrm{P}_{0}$ between 1600 to $2500 \mathrm{~Hz}$ to below $116.00 \mathrm{~dB}$ and keep the highest structural energy density level below $126.0 \mathrm{~dB}$.

The design objective is to minimize the mass cost, which is related to both thickness and damping design variables. First of all, the vehicle mass cost is directly contributed by the thickness design variables and is expressed by

$$
f_{1}=\sum_{N E} \int_{\Omega_{i}} \rho_{i} h_{i} d \Omega
$$

with $N E$ as the total number of the finite elements, $\rho_{\mathrm{i}}$ the mass density of the $i$-th finite element and $h_{\mathrm{i}}$ the thickness of the $i$-th finite element. 
The mass cost related to damping design variables is determined by the ways of damping adjustment: Free Layer Damping or Constrained Layer Damping treatment [43]. The former is also called surface damping treatment in which a "high-loss" material (usually rubbery, asphalt, vinyl) is sprayed on, brushed on, or adhesively bonded to the panel surface as shown in Fig. 15(a). The latter is a treatment that a relatively thin layer of damping material is sandwiched between the structure and a stiff but thin cover plate as retaining layer, such as aluminum, steel or sheet metal (Fig. 15(b)). While the constrained layer damping treatment has better damping enhancement than the free layer damping, the free layer damping is much easier and more practical in engineering applications. For example, the asphalt sheets have been used for over 50 years in automotive, naval and aerospace industry and are still widely used because of the low material cost. On the other hand, the constrained layer damping needs additional aluminum layer for the damping treatment, and it needs to be extruded, slit and cut into preferred shapes, which requires more material and labor cost involved than the free layer damping treatment.

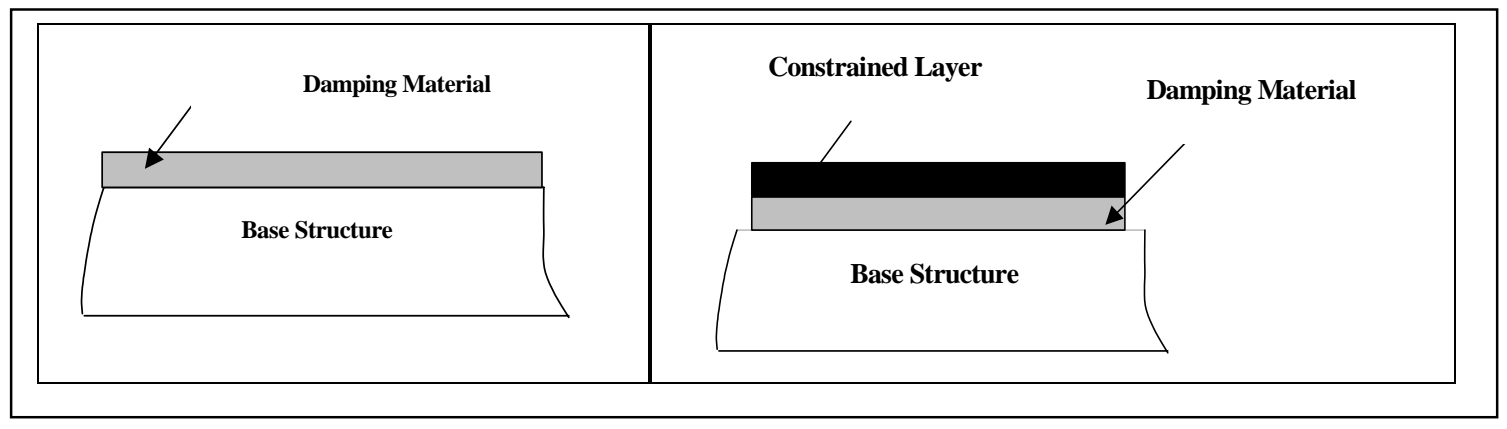

(a) Free Layer Damping

(b) Constrained Layr Damping

Figure 15 Damping Treatments

Some researches have been carried out in the prediction of the mechanical properties of different surface damping treatments, such as the RKU analysis developed by Ross, Ungar and Kerwin [44]. It is shown that the properties of the laminated material are functions of the thickness and material properties of each layer. However, the damping materials are usually provided in standard products from manufacturers, whose thicknesses are not adjustable. Moreover, the material properties of the mostly used damping materials, including the mass density, Young's modulus, Poisson's ratio and hysteresis damping factor are unknown or largely variant in most cases. As a result, it is difficult to analytically model the damping mass directly related to the total vehicle mass cost. However, the mass contributed by the damping adjustment is proportional to the amount of damping adjustment and the area on which the adjustment is applied. To simplify the problem in this study, the mass of the damping material adjustment is assumed to be linear in terms of the damping increment and the surface area as

$$
f_{2}=\sum_{N E} \int_{\Omega_{i}}\left(\eta_{i}-\eta_{i}^{o r i}\right) d \Omega
$$

where $\eta_{i}^{\text {ori }}$ is the original damping on the $i$-th finite element, and $\eta_{i}$ is the proposed damping computed by the design optimization algorithm.

Due to the existence of two different objective functions in Eqs. (64) and (65), a multi-objective design optimization needs to be carried out instead of a single-objective design optimization. In this paper, the multiobjective design optimization problem is transformed into a single-objective design optimization problem, where the Weighted Objective Method is used to convert the multi-objective functions into a single-objective function by a weighted sum as

$$
f=w_{1} f_{1}+w_{2} f_{2}
$$

where $w_{1}$ and $w_{2}$ are called "weighting coefficients" and they have the following relationship

$$
w_{1}+w_{2}=1
$$


The optimization results of Eq. (66) are infinite subject to the different combination of weighting coefficients. If the coefficients are selected at discrete set of points, a corresponding discrete set of optimization results will be obtained, which is called the Pareto Optimal set. The design engineers are expected to pick the values of the results from the Pareto Optimal set, based on different design situations. For example, if the contribution of material damping to vehicle mass can be ignored as in Chapter 6, i.e., $w_{1}=1$, and $w_{2}=0$, the optimization will be simply carried out to reduce the vehicle mass using thickness design variables; On the contrary, if the damping adjustment is not preferred compared to the thickness adjustment, i.e., $w_{1}=0$, and $w_{2}=1$, the design optimization will be carried out to reduce the damping material adjustment without considering the contribution of thickness adjustment to vehicle mass. If the contribution of material damping to vehicle mass is considered as important as the contribution of metal thickness to vehicle mass, i.e., $w_{1}=w_{2}=0.5$, the design optimization will be carried out to search optimum to reduce both the damping and thickness adjustment under certain constraints.

Using the Weighted Objective Method, the design optimization can be carried out on the parametric design variables, including the material thickness and hysteresis damping factor of all the 11 substructures of the undersea vehicle morel. As a result, the design optimization problem is formulated as

Objective: Minimize the weighted objective function

$$
f=w_{1} \frac{f_{1}}{F_{1}}+w_{2} \frac{f_{2}}{F_{2}} \quad\left(0.5<f_{1} / F_{1}<1.5,0<f_{2} / F_{2}<1,0<w_{1}<1,0<w_{2}<1\right)
$$

Constraints: Subject to two sets of constraints

(1) Reduce Noise Level at far-field point $\mathrm{P}_{0}$ in $1600-2500 \mathrm{~Hz}$ to $116.0 \mathrm{~dB}$

(2) Keep the highest structural energy density level in 1600-2500 Hz below $126.0 \mathrm{~dB}$

\section{Parametric Design Variables:}

11 Panel Thickness ( $\pm 50 \%$ Design Change Allowed)

11 Panel Damping (200\% Design Change Allowed)

where in Eq. (68), the objective functions $f_{1}$ and $f_{2}$ are normalized with respect to the maximum allowable mass and damping cost changes $F_{1}$ and $F_{2}$, which are distances between the upper and lower limits of the thickness and damping design space.

The design optimization is carried out for the different combination of weighting coefficients $w_{1}$ and $w_{2}$ as shown in Table 4 and DOT (Design Optimization Tool) is used as optimization program where SQP (Sequential Quadratic Programming) is chosen as the optimization algorithm. The Pareto Optimal results are obtained and listed in Table 4, while the Pareto Optimal set is plotted in Fig. 16, which shows that the weighted objection function is convex, and the reduction of one objective function needs to compromise the other.

Table 4 Pareto Optimal Results for Different Weighting Coefficients

\begin{tabular}{llllll}
\hline$w_{1}$ & 1.0 & 0.75 & 0.5 & 0.25 & 0.0 \\
\hline$w_{2}$ & 0.0 & 0.25 & 0.5 & 0.75 & 1.0 \\
\hline$f_{l} / F_{l}$ & 0.573 & 0.590 & 0.608 & 0.633 & 0.665 \\
\hline$f_{l}(\mathrm{Kg})$ & 181.3 & 186.5 & 192.4 & 200.3 & 210.5 \\
\hline$f_{2} / F_{2}$ & 1.000 & 0.714 & 0.642 & 0.604 & 0.575 \\
\hline
\end{tabular}




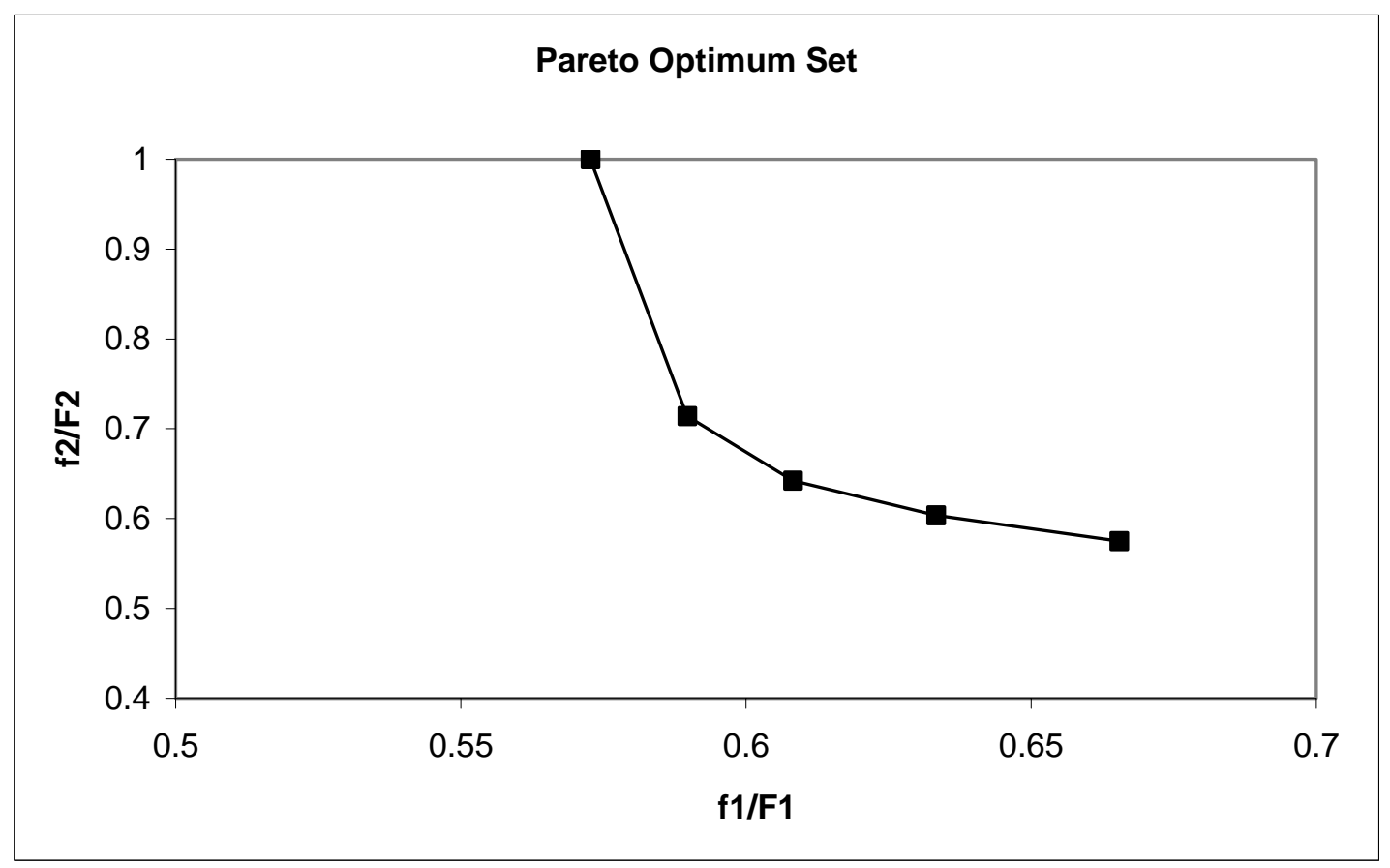

Figure 16 Patero Optimal Set of Multi-Objective Optimization

To illustrate the optimization results, the history of the vehicle mass cost for the case when $w_{1}=1$ and $w_{2}=0$ is plotted in Fig. 17, from which we can see that the optimization is converged after 4 iterations, and at the end of the optimization, the vehicle mass is reduced from $306 \mathrm{Kg}$ to $172 \mathrm{Kg}$, a $134 \mathrm{Kg}$ reduction. Such large amount of mass reduction is achieved by maximizing use of damping material, which is shown in Fig. 18 where all the damping design variables increase to the maximum allowable value. Compared with the damping design variables, the history of the thickness design variables illustrated in Fig. 19 shows that all the thickness design variables decrease to the minimum allowable value to reduce the mass, except the thickness of substructure 5, which reduces by a certain amount but does not hit the lower boundary of the design space to help keep the design constraints satisfied. 


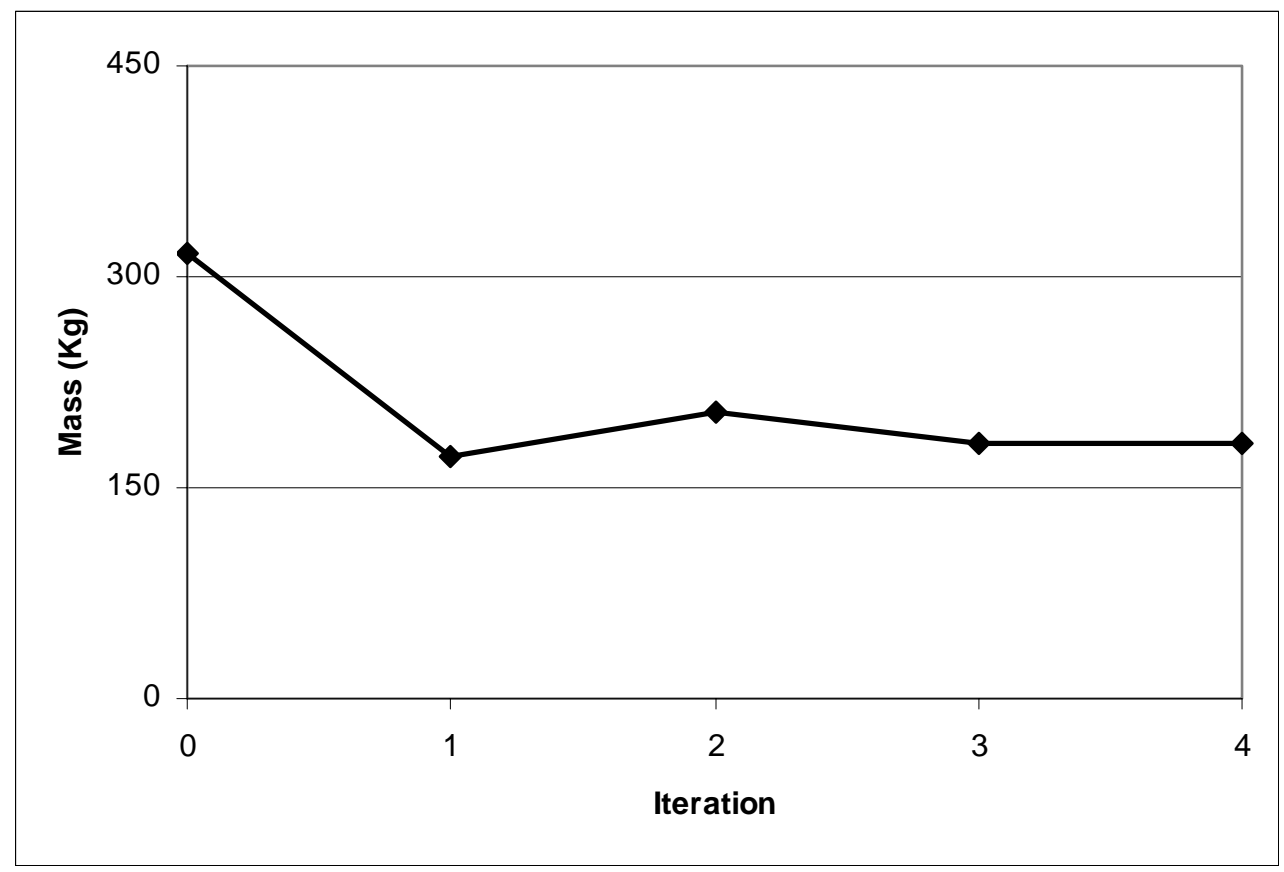

Figure 17 History of Vehicle Mass Cost Function

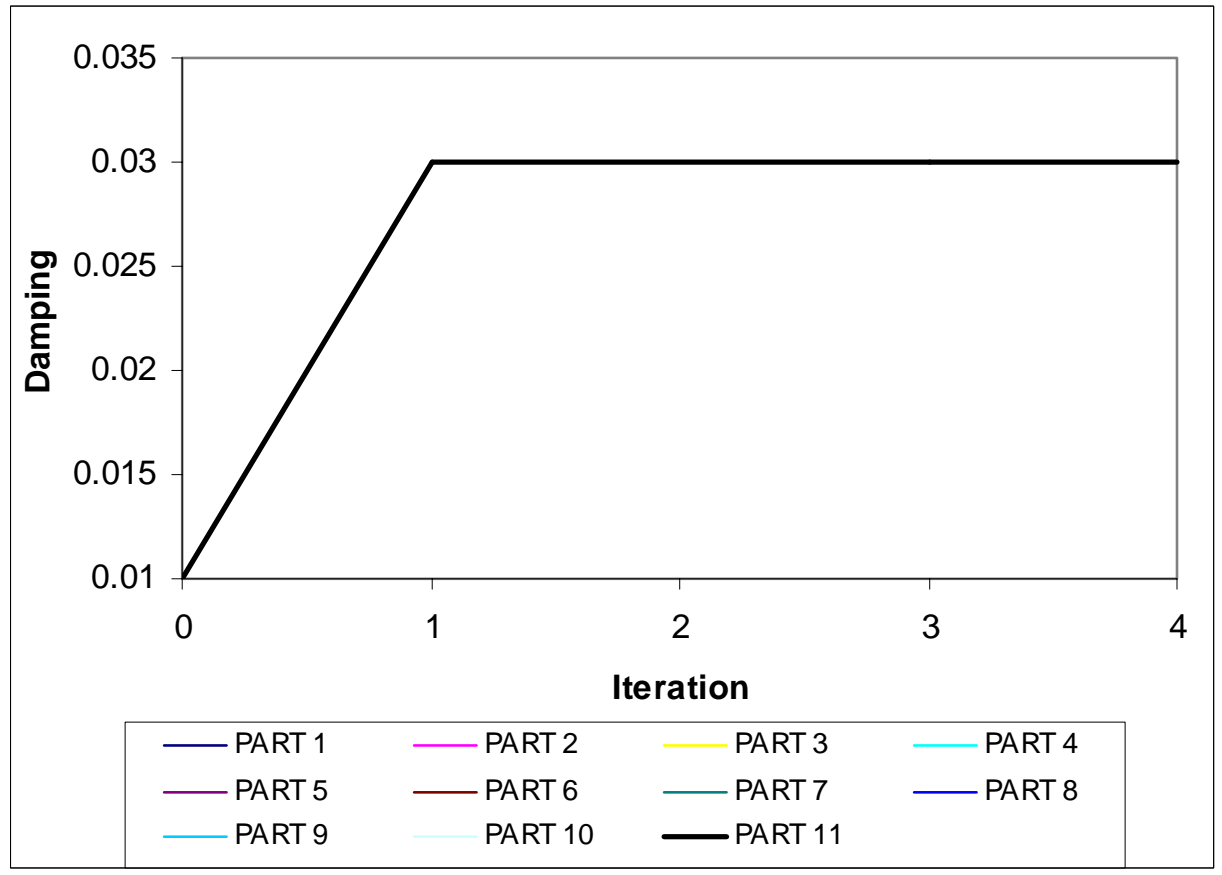

Figure 18 History of Damping Design Variables 


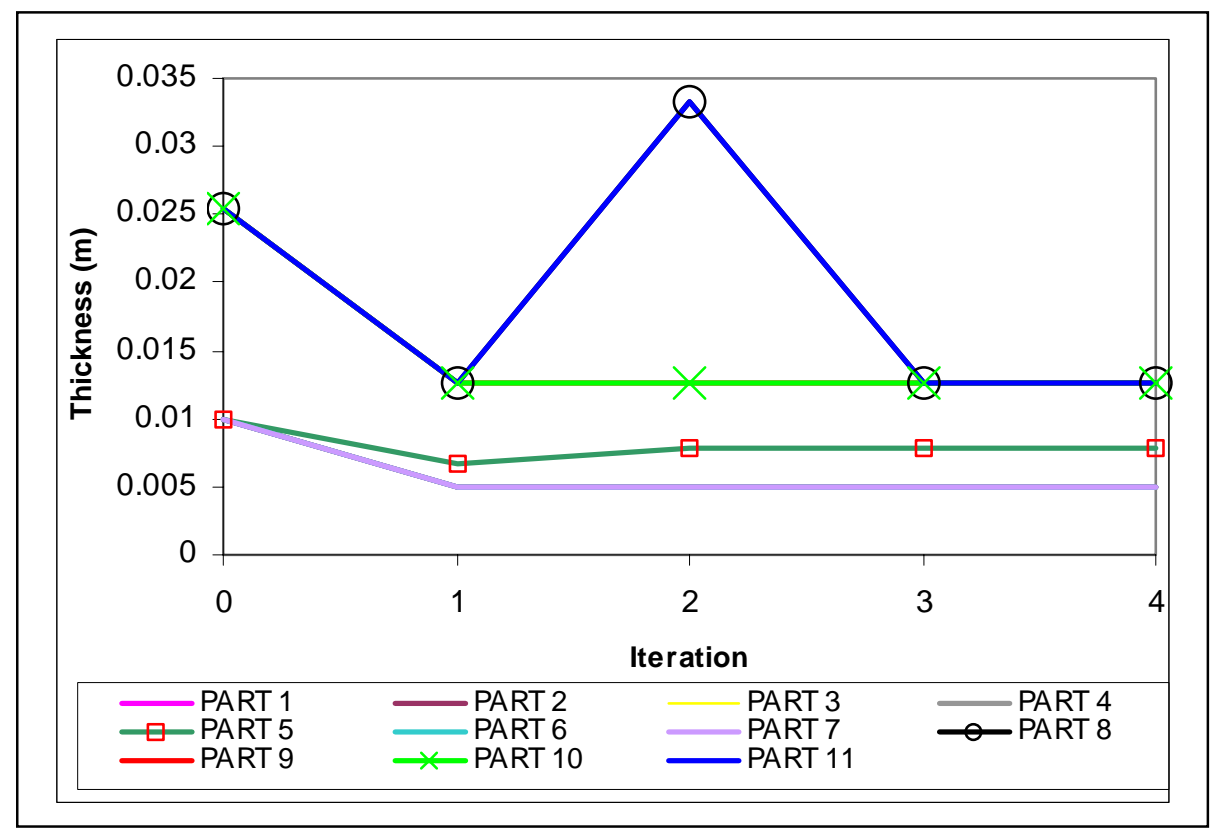

Figure 19 History of Thickness Design Variables

The comparison of the design constraints for the far-field acoustic pressure at the original and optimum design is plotted in Fig. 20, which shows that the highest radiation noise is reduced from $118.9 \mathrm{~dB}$ to $116.0 \mathrm{~dB}$, equivalent to $28.4 \%$ reduction in magnitude. At the same time, Figure 21 compares the design constraints on the highest structural energy level at the original and optimum design, which shows that although the design changes causes reduction of the far-field radiation noise, the highest structural energy level is still kept below $126.0 \mathrm{~dB}$, the level of the original design.

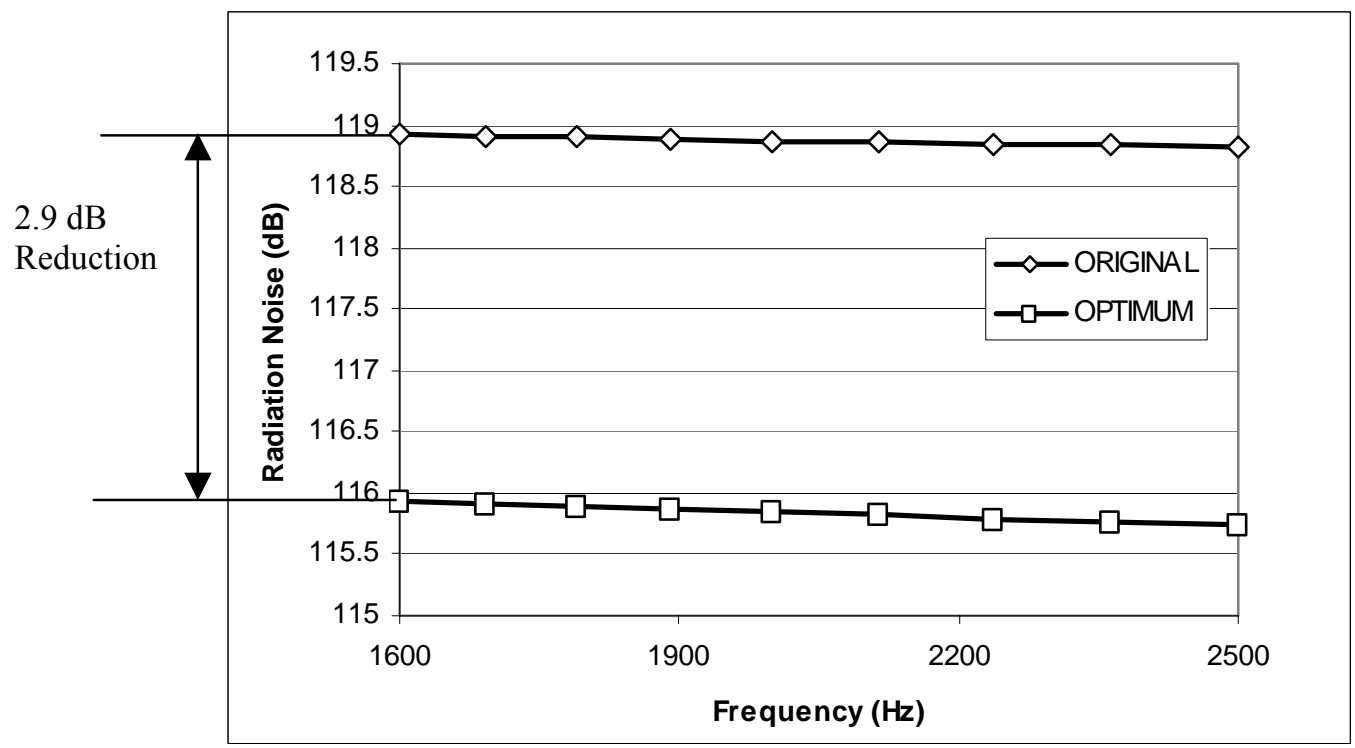

Figure 20 History of Acoustic Design Constraints 


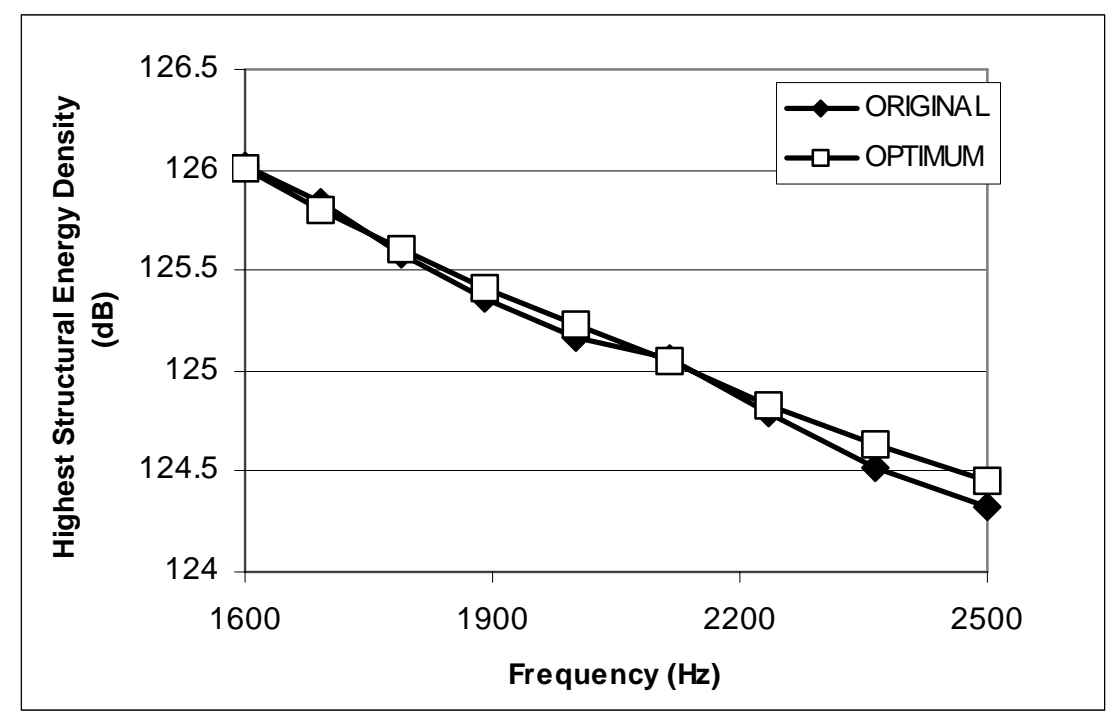

Figure 21 History of Structural Design Constraints

The optimum design results of the thickness and damping design variables are listed in Table 5, which provides design engineers guidance for design modification under different requirements. If the contribution of damping material to vehicle mass is ignored, then all the damping design variables will increase to the upper bound in the design space and the thickness design variables will reduce correspondingly to reduce the vehicle mass without violating the design constraints. Once the contribution of damping material to vehicle mass is considered in the total vehicle mass function, and with the increase of the weighting coefficient $w_{2}$, most of the damping design variables need to decrease to reduce the mass contribution from damping adjustment, while the thickness design variables needs to increase correspondingly to meet the design constraints. If the contribution of metal thickness to vehicle mass is ignored, most of the damping design variables will reduce to the lower bound in the design space except the damping in substructures 5 and 6, which stays at the upper bound to satisfy the design constraints. On the contrary, all the thickness decreases to the lower bound in the design space, except that the thickness of substructures 8 and 11 increases to the upper bound in the design space and the thickness of substructure 5 decreases to a certain level above the lower bound in the design space to help satisfy the design constraints. 
Table 5 Thickness and Damping Design Results for Pareto Optimal Set

\begin{tabular}{ccccccc}
\hline & $\begin{array}{c}\text { Original } \\
\text { Design }\end{array}$ & & & $\begin{array}{c}\text { Optimum } \\
\text { Design }\end{array}$ & & \\
\hline & & $w_{1}=1.0$ & $w_{1}=0.75$ & $w_{1}=0.5$ & $w_{1}=0.25$ & $w_{1}=0.0$ \\
& & $w_{2}=0.0$ & $w_{2}=0.25$ & $w_{2}=0.75$ & $w_{2}=1.0$ \\
\hline$h_{1}$ & $1.00 \mathrm{E}-02$ & $5.00 \mathrm{E}-03$ & $5.00 \mathrm{E}-03$ & $5.00 \mathrm{E}-03$ & $5.00 \mathrm{E}-03$ & $5.00 \mathrm{E}-03$ \\
\hline$h_{2}$ & $1.00 \mathrm{E}-02$ & $5.00 \mathrm{E}-03$ & $5.00 \mathrm{E}-03$ & $5.00 \mathrm{E}-03$ & $5.00 \mathrm{E}-03$ & $5.00 \mathrm{E}-03$ \\
\hline$h_{3}$ & $1.00 \mathrm{E}-02$ & $5.00 \mathrm{E}-03$ & $5.00 \mathrm{E}-03$ & $5.00 \mathrm{E}-03$ & $5.00 \mathrm{E}-03$ & $5.00 \mathrm{E}-03$ \\
\hline$h_{4}$ & $1.00 \mathrm{E}-02$ & $5.00 \mathrm{E}-03$ & $5.00 \mathrm{E}-03$ & $5.00 \mathrm{E}-03$ & $5.00 \mathrm{E}-03$ & $5.00 \mathrm{E}-03$ \\
\hline$h_{5}$ & $1.00 \mathrm{E}-02$ & $7.85 \mathrm{E}-03$ & $7.93 \mathrm{E}-03$ & $7.88 \mathrm{E}-03$ & $7.87 \mathrm{E}-03$ & $7.89 \mathrm{E}-03$ \\
\hline$h_{6}$ & $1.00 \mathrm{E}-02$ & $5.00 \mathrm{E}-03$ & $5.00 \mathrm{E}-03$ & $5.00 \mathrm{E}-03$ & $5.00 \mathrm{E}-03$ & $5.00 \mathrm{E}-03$ \\
\hline$h_{7}$ & $1.00 \mathrm{E}-02$ & $5.00 \mathrm{E}-03$ & $5.00 \mathrm{E}-03$ & $5.00 \mathrm{E}-03$ & $5.00 \mathrm{E}-03$ & $5.00 \mathrm{E}-03$ \\
\hline$h_{8}$ & $2.54 \mathrm{E}-02$ & $1.27 \mathrm{E}-02$ & $2.09 \mathrm{E}-02$ & $3.21 \mathrm{E}-02$ & $2.93 \mathrm{E}-02$ & $3.81 \mathrm{E}-02$ \\
\hline$h_{9}$ & $2.54 \mathrm{E}-02$ & $1.27 \mathrm{E}-02$ & $1.27 \mathrm{E}-02$ & $1.27 \mathrm{E}-02$ & $1.27 \mathrm{E}-02$ & $1.27 \mathrm{E}-02$ \\
\hline$h_{10}$ & $2.54 \mathrm{E}-02$ & $1.27 \mathrm{E}-02$ & $1.27 \mathrm{E}-02$ & $1.27 \mathrm{E}-02$ & $1.27 \mathrm{E}-02$ & $1.27 \mathrm{E}-02$ \\
\hline$h_{11}$ & $2.54 \mathrm{E}-02$ & $1.27 \mathrm{E}-02$ & $1.27 \mathrm{E}-02$ & $1.27 \mathrm{E}-02$ & $2.88 \mathrm{E}-02$ & $3.81 \mathrm{E}-02$ \\
\hline$\eta_{1}$ & $1.00 \mathrm{E}-02$ & $3.00 \mathrm{E}-02$ & $1.46 \mathrm{E}-02$ & $1.02 \mathrm{E}-02$ & $1.00 \mathrm{E}-02$ & $1.00 \mathrm{E}-02$ \\
\hline$\eta_{2}$ & $1.00 \mathrm{E}-02$ & $3.00 \mathrm{E}-02$ & $1.80 \mathrm{E}-02$ & $1.02 \mathrm{E}-02$ & $1.00 \mathrm{E}-02$ & $1.00 \mathrm{E}-02$ \\
\hline$\eta_{3}$ & $1.00 \mathrm{E}-02$ & $3.00 \mathrm{E}-02$ & $1.95 \mathrm{E}-02$ & $1.03 \mathrm{E}-02$ & $1.00 \mathrm{E}-02$ & $1.00 \mathrm{E}-02$ \\
\hline$\eta_{4}$ & $1.00 \mathrm{E}-02$ & $3.00 \mathrm{E}-02$ & $2.04 \mathrm{E}-02$ & $2.44 \mathrm{E}-02$ & $2.39 \mathrm{E}-02$ & $2.64 \mathrm{E}-02$ \\
\hline$\eta_{5}$ & $1.00 \mathrm{E}-02$ & $3.00 \mathrm{E}-02$ & $3.00 \mathrm{E}-02$ & $3.00 \mathrm{E}-02$ & $3.00 \mathrm{E}-02$ & $3.00 \mathrm{E}-02$ \\
\hline$\eta_{6}$ & $1.00 \mathrm{E}-02$ & $3.00 \mathrm{E}-02$ & $3.00 \mathrm{E}-02$ & $3.00 \mathrm{E}-02$ & $3.00 \mathrm{E}-02$ & $3.00 \mathrm{E}-02$ \\
\hline$\eta_{7}$ & $1.00 \mathrm{E}-02$ & $3.00 \mathrm{E}-02$ & $3.00 \mathrm{E}-02$ & $2.78 \mathrm{E}-02$ & $1.65 \mathrm{E}-02$ & $1.16 \mathrm{E}-02$ \\
\hline$\eta_{8}$ & $1.00 \mathrm{E}-02$ & $3.00 \mathrm{E}-02$ & $1.00 \mathrm{E}-02$ & $1.00 \mathrm{E}-02$ & $1.00 \mathrm{E}-02$ & $1.00 \mathrm{E}-02$ \\
\hline$\eta_{9}$ & $1.00 \mathrm{E}-02$ & $3.00 \mathrm{E}-02$ & $3.00 \mathrm{E}-02$ & $3.00 \mathrm{E}-02$ & $3.00 \mathrm{E}-02$ & $1.06 \mathrm{E}-02$ \\
\hline$\eta_{10}$ & $1.00 \mathrm{E}-02$ & $3.00 \mathrm{E}-02$ & $3.00 \mathrm{E}-02$ & $3.00 \mathrm{E}-02$ & $3.00 \mathrm{E}-02$ & $2.32 \mathrm{E}-02$ \\
\hline$\eta_{11}$ & $1.00 \mathrm{E}-02$ & $3.00 \mathrm{E}-02$ & $1.00 \mathrm{E}-02$ & $1.00 \mathrm{E}-02$ & $1.00 \mathrm{E}-02$ & $1.00 \mathrm{E}-02$ \\
\hline & & & & & & \\
\hline
\end{tabular}




\section{Conclusion}

A continuum DSA of high frequency radiation problems using EFEM and EBEM is formulated and presented. It is successfully applied in the design of automotive and naval structures to search for the best material layout to achieve lowest noise level at high frequency. The DSA method presents excellent numerical accuracy compared with the finite difference results in the numerical examples of the noise radiation of automotive and naval structures in light and dense fluids, and the DSA information has been used in a multi-objective optimization of naval structure to achieve the optimum design where the structural mass is minimized and the high frequency underwater noise radiation is reduced.

\section{Acknowledgement}

This research is supported by the Automotive Research Center sponsored by the US Army TARDEC under contract DAAE07-94-C-R094. The authors gratefully acknowledge this support.

\section{References}

${ }^{1}$ Zienkiewicz, O.C., Taylor, R.L., The Finite Element Method: Basic Formulation and Linear Problems (4th edition), McGraw-Hill Book Company Limited, England, 1989.

${ }^{2}$ Hughes, T. J. R., The Finite Element Method, Prentice-Hall, Englewood Cliffs, NJ, 1987.

${ }^{3}$ Bathe, K.J., Finite Element Procedures, Englewood Cliffs, NJ; Prentice Hall, 1996.

${ }^{4}$ Brebbia, C. A., and Dominguez, J. Boundary Elements An Introductory Course, Computational Mechanics Publications, Boston, 1989.

${ }^{5}$ Banerjee, P. K. and Butterfield, R., Boundary Element Methods in Engineering Science, McGraw-Hill Book Company, New York, 1981.

${ }^{6}$ Kythe, P. K., Introduction to Boundary Element Methods, CRS Press, Florida, 1995.

${ }^{7}$ Atalla, N., Bernhard, R. J., "Review of Numerical Solutions for Low-Frequency Structural-Acoustic Problems", Applied Acoustics, 43 (3): 271-294, 1994.

${ }^{8}$ Lyon, R., Eichler, E., "Random Vibration of Connected Structures", Journal of Acoustic Society of America, Vol. 36, No. 7, pp 1344-1354, July 1964.

${ }^{9}$ Lyon, R., Statistical Energy Analysis of Dynamical Systems: Theory and Application, The MIT Press, Cambridge, MA, 1975.

${ }^{10}$ Lyon, R., and DeJong, R. G., Theory and Application of Statistical Energy Analysis (2nd Edition), ButterworthHeinemann, Boston, MA, 1995.

${ }^{11}$ Nefske, D. J., Wolf, J. A., and Howell, L. J., "Structural-acoustic finite element analysis of the automobile passenger compartment: A review of current practice," Journal of Sound and Vibration, 80, pp. 247-266, 1982.

${ }^{12}$ Wohlever J., and Bernhard, R. J., "Mechanical Energy Flow Models of Rods and Beams", Journal of Sound and Vibration 153(1) 1-19, 1992.

${ }^{13}$ Bouthier, O. M., and Bernhard, R. J., "Models of Space-Averaged Energetics of Plates", AIAA Journal 30(3) 616-623, 1992.

${ }^{14}$ Bouthier, O. M., "Energetics of Vibrating Systems", Ph.D. Thesis, Purdue University, 1992.

${ }^{15}$ Bouthier, O. M. and Bernhard, R. J., "Simple-Models of the Energetics of Transversely Vibrating Plates", Journal of Sound and Vibration 182(1) 149-166, 1995.

${ }^{16}$ Cho, P. E., and Bernhard, R. J., "Energy Flow Analysis of Coupled Beams”, Journal of Sound and Vibration 211(4) 593605, 1998.

${ }^{17}$ Cho, P. E., "Energy Flow Analysis of Coupled Structures", Ph.D. Thesis, Purdue University, 1993.

${ }^{18}$ Bernhard, R. J. and Huff, J. E., "Structural-Acoustic Design at High Frequency Using the Energy Finite Element Method", Journal of Vibration and Acoustics 121(3) 295-301, 1999.

${ }^{19}$ Zhang, W., Wang, A., Vlahopoulos, N., "An Alternative Energy Finite Element Formulation Based on Incoherent Orthogonal Waves and Its Validation for Marine Structures", Finite Elements in Analysis and Design, Volume 38, Issue 12, pp.1095-1113, October 2002.

${ }^{20}$ Bitsie, F., "The Structural-Acoustic Energy Finite Element Method and Energy Boundary Element Method", Ph.D. Thesis, Purdue University, 1996.

${ }^{21}$ Langley, R. S., and Heron, K. H., "Elastic Wave Transmission through Plate/Beam Junctions", Journal of Sound and Vibration 143(2) 241-253, 1990.

${ }^{22}$ Zhang, W., Wang, A., Vlahopoulos, N., "High Frequency Vibration Analysis of Thin Elastic Plates Under Heavy Fluid Loading by an Energy Finite Element Formulation", Journal of Sound and Vibration, 263 (1): 21-46 MAY 222003.

${ }^{23}$ Vlahopoulos N., "A Numerical Structure-Borne Noise Prediction Scheme Based on the Boundary-Element Method, With a New Formulation for the Singular-Integrals”, Computers \& Structures, 50 (1): 97-109 JAN 31994. 
${ }^{24}$ Zhang, W., Wang, A., Vlahopoulos, N., "Validation of the EFEA Method through Correlation with Conventional FEA and SEA Results", 2001 SAE Noise and Vibration Conference, SAE Paper_No. 2001-01-1618, 2001.

${ }^{25}$ Wang, S., "Theory and Applications of a Simplified Energy Finite Element Method and Its Similarity to SEA", Noise Control Engineering Journal 50 (2): 63-72 MAR-APR 2002.

${ }^{26}$ Wang, A., Vlahopoulos, N., Wu, K., "Development of An Energy Boundary Element Formulation of Sound Radiation at High Frequency", in press, Journal of Sound and Vibration, 2004

${ }^{27}$ Choi, K. K., and Lee, J. H., "Sizing Design Sensitivity Analysis of Dynamic Frequency Response of Vibrating Structures," ASME Journal of Mechanical Design, 114, pp. 166-173, 1992.

${ }^{28}$ Choi, K. K., Shim, I., and Wang, S., "Design Sensitivity Analysis of Structure-Induced Noise and Vibration", Journal of Vibration and Acoustics 119(2) 173-179, 1997.

${ }^{29}$ Kim, N. H., Dong, J., Choi, K. K., Vlahopoulos, N., Ma, Z. D., Castanier, M., and Pierre, C. "Design Sensitivity Analysis for Sequential Structural-Acoustic Problems", Journal of Sound and Vibration, 263 (3): 569-591 JUN 52003.

${ }^{30}$ Dong, J., Choi, K. K., Kim, N. H., "Design Optimization of Structural-Acoustic Problem Using FEA-BEA with Adjoint Variable Method", in press, ASME Journal of Mechanical Design, 2004.

${ }^{31}$ Ma, Z. D., and Hagiwara, I., "Sensitivity analysis-method for coupled acoustic-structural systems Part 1: modal sensitivities," AIAA Journal, 29, pp. 1787-1795, 1991.

${ }^{32} \mathrm{Ma}$, Z. D., and Hagiwara, I., "Sensitivity analysis-method for coupled acoustic-structural systems Part 2: direct frequencyresponse and its sensitivities," AIAA Journal, 29, pp. 1796-1801, 1991.

${ }^{33} \mathrm{Ma}$, Z. D., and Hagiwara, I., "Development of Eigenmode and Frequency Response Sensitivity Analysis Methods for Coupled Acoustic-Structural Systems", JSME International Journal, Series III, Vol. 35, No. 2, pp 229-235, 1995.

${ }^{34}$ Kane, J. H., Mao, S., and Everstine, G. C., "Boundary element formulation for acoustic shape sensitivity analysis", Journal of the Acoustical Society of America, 90, pp. 561-573, 1991.

${ }^{35}$ Cunefare K. A., and Koopman, G. H., "Acoustic design sensitivity for structural radiators," Journal of Vibration and Acoustics, 114, pp. 179-186, 1992.

${ }^{36}$ Koo, B. U., "Shape Design Sensitivity Analysis of Acoustic Problems Using a Boundary Element Method", Computers and Structures 65(5) 713-719, 1997.

${ }^{37}$ Matsumoto, T., Tanaka, M., and Yamada, Y., 1995, "Design sensitivity analysis of steady-state acoustic problems using boundary integral equation formulation," JSME International Journal Series C, 38, pp. 9-16.

${ }^{38}$ Smith, D. C., and Bernhard, R. J., "Computation of Acoustic Shape Shape Design Sensitivity Using A Boundary Element Method", Journal of Vibration and Acoustics-Transactions of The ASME, 114 (1): 127-132 JAN 1992.

${ }^{39}$ Kim, N. H., Dong, J., Choi, K. K., "Energy Flow Analysis and Design Sensitivity Analysis of Structural-Acoustic Problems at High Frequency", Journal of Sound and Vibration, 269 (1-2): 213-250, 2004.

${ }^{40}$ Choi, K. K., Dong, J., Vlahopoulos, N., Wang, A., Zhang, W., "Parametric Design Sensitivity Analysis of High Frequency Structural-Acoustic Problems Using Energy Finite Element Method", ASME 2003 Design Engineering Technical Conferences and Computers and Information in Engineering Conference, DETC/DAC-48753, Sep. 02 - Sep. 06, 2003, Chicago, IL.

${ }^{41}$ Dong, J., Choi, K. K., Wang, A., Zhang, W., Vlahopoulos, N., "Parametric Design Sensitivity Analysis of High Frequency Structural-Acoustic Problems Using Energy Finite Element Method", accepted, International Journal for Numerical Methods in Engineering, 2004.

${ }^{42}$ Leppington, F. G., Broadbent, E. G., F.R.S., and Heron, K. H., "The Acoustic Radiation Efficiency of Rectangular Panels", Proc. R. Soc. Lond., A 382, 245-271, 1982.

${ }^{43}$ Nashif, A. D., Jones, D. I. G., and Henderson, J. P., Vibration Damping, John Wiley \& Sons, Inc, 1985.

${ }^{44}$ Ross, D., Ungar. E. E., and Kerwin, E. M. Jr., "Damping of Plate Flexural Vibrations by Means of Viscoelastic Laminates", Structural Damping (papers presented at a colloqium on structural damping held at the ASME annual meeting in Atlantic City, N.J., in December 1959), ASME, New York, pp 49-88, 1959. 